Pacific

Journal of

Mathematics

\title{
CMC CAPILLARY SURFACES AT REENTRANT CORNERS
}

MaRia ATHANASSENAS AND KIRK LANCASTER 


\title{
CMC CAPILLARY SURFACES AT REENTRANT CORNERS
}

\author{
Maria Athanassenas AND KiRK LANCASTER
}

\begin{abstract}
For a capillary graph in a vertical cylinder $\Omega \times \mathbb{R} \subset \mathbb{R}^{3}$, the existence of a reentrant corner $P \in \partial \Omega$ makes the determination of the continuity at $P$ (or the behavior of the radial limits at $P$ ) of the solution problematic. Since continuity is the necessary consequence of the existence of a "central fan" of radial limits under certain conditions, the determination of necessary and sufficient conditions for the existence of a central fan is a very important open question in the mathematical theory of capillarity. Examples by Finn and Shi suggest that "central fans" may be very rare in the sense that arbitrarily small perturbations can eliminate them. In this note we obtain examples of capillary graphs (with zero mean curvature), each of which is continuous or has a central fan at a reentrant corner.
\end{abstract}

\section{Introduction}

The nonparametric capillary problem in a cylinder $\Omega \times \mathbb{R}$ over a piecewise smooth domain $\Omega$ with corners at points $A_{1}, \ldots, A_{n} \in \partial \Omega$ and gravitational constant $\kappa$ consists of finding the solution(s) $h \in C^{2}(\Omega) \cap C^{1}\left(\bar{\Omega} \backslash\left\{A_{1}, \ldots, A_{n}\right\}\right)$ of the Dirichlet problem

$$
\begin{aligned}
\operatorname{div}(T h)=\kappa h+\lambda & \text { in } \Omega, \\
T h \cdot \boldsymbol{v}=\cos (\gamma) & \text { on } \partial \Omega \backslash\left\{A_{1}, \ldots, A_{n}\right\},
\end{aligned}
$$

where $\boldsymbol{v}$ is the outer unit normal to $\partial \Omega$ and

$$
T h=\frac{\nabla h}{\sqrt{1+|\nabla h|^{2}}} .
$$

An extremely interesting and important question about nonparametric capillary surfaces is that of determining necessary and sufficient conditions for the continuity at a corner $P$ of a solution $h$ of (1). As a solution of a boundary value problem for a quasilinear elliptic equation with positive genre $(g=2)$, the possibility of obtaining such conditions should seem remote; solutions of equations like (1a) can behave near a point on the boundary in significantly different ways than can solutions of

MSC2000: primary 76B45; secondary 53A10, 76B03.

Keywords: minimal surface, capillary graph, Riemann-Hilbert problem. 
equations such as Laplace's equation or a Poisson equation, as illustrated by [Jin and Lancaster 1999] and [Serrin 1969].

Let us assume $\Omega$ is a domain in $\mathbb{R}^{2}$ whose boundary has a corner of size $2 \alpha$ at a point, which we may temporarily take to be the origin $O=(0,0)$ for convenience; we may assume the domain is oriented so that the rays $\theta=\alpha$ and $\theta=-\alpha$ are tangent to $\partial \Omega$ at $O$ and so that the directions $\theta \in(-\alpha, \alpha)$ are the interior directions to $\Omega$ from $O$, where $(r, \theta)$ denotes polar coordinates about $O$. We may parametrize $\partial \Omega$ near $O$ by $(x(s), y(s))$ so that $(x(0), y(0))=(0,0)$ and $y(s)>0$ if $s>0$ but $y(s)<0$ if $s<0$. Let us also suppose that the contact angle $\gamma=\gamma(x, y)$ might depend on $(x, y) \in \partial \Omega$ and assume the limits

$$
\gamma_{1}=\lim _{s \downarrow 0} \gamma(x(s), y(s)) \quad \text { and } \quad \gamma_{2}=\lim _{s \uparrow 0} \gamma(x(s), y(s))
$$

both exist.

The study of capillary surfaces in wedge domains can be tricky, as illustrated by [Keller et al. 1991] and [Vreeburg 1990]. Concus and Finn [1991] mentioned that [Vreeburg 1990] was incorrect; we note that its argument is invalid since it does not distinguish between mean curvature and Gauss curvature and since it ignores [Tam 1986]. Vreeburg fails to consider the possibility of a jump discontinuity in the trace of the capillary surface on the boundary cylinder; see Section 2 and [Lancaster and Siegel 1996b]. Concus and Finn [1996b] noted that [Keller et al. 1991] was incorrect. Keller, King and Merchant [1991] argued that the only ruled minimal surfaces in $\mathbb{R}^{3}$ were planes, ignoring helicoids, and seemed to be confused about the notion of principal curvature. As with Vreeburg, they failed to consider the possibility of bounded, discontinuous (at the corner) capillary surfaces when $\gamma_{1} \neq \gamma_{2}$. Concus and Finn did consider this important possibility; as part of their investigation of these capillary surfaces, they discussed the question of continuity at a corner and in 1992 formulated the following conjecture for convex corners; see for example [Shi 2006].

Concus-Finn Conjecture. Suppose $\alpha \in(0, \pi / 2), \gamma_{1}, \gamma_{2} \in(0, \pi)$, and $\left|\gamma_{1}-\gamma_{2}\right|>$ $\pi-2 \alpha$. Then a solution of (1) must be discontinuous at $O$.

Concus and Finn [1996a] and Lancaster and Siegel [1996b; 1996a] studied the behavior near $O$ of capillary surfaces over domains with a corner at $O$ whose contact angles might have differing limits at $O$; see also [Concus and Finn 1994; Concus et al. 1992].

For convex corners $(2 \alpha \leq \pi)$, the continuity or discontinuity of a solution of (1) at such a corner is completely determined by "local" geometric information, that is, $\gamma_{1}, \gamma_{2}$, and $\alpha$. At nonconvex corners $(2 \alpha>\pi)$, the task of finding necessary and sufficient conditions for continuity is much more difficult, and local information (that is, $\gamma_{1}, \gamma_{2}$ and $\alpha$ ) is not necessarily sufficient to determine continuity. 
Our first goal is to provide an overview of results related to the continuity of a solution $h$ of (1) and to introduce a problem, the "central fan question", which we consider to be the most important open problem in the mathematical theory of capillary surfaces; we do this in Section 2. Our second goal is to demonstrate that for a range of piecewise constant contact angle data $\gamma$, there exists a domain $\Omega$ with a reentrant corner at $P$ such that either the solution $h$ of the capillary problem with $\kappa=0$ is continuous at $P$ or the radial limits of $h$ at $P$ exhibit a central fan.

Our examples complement [Shi 2006, Example 6.6], which is a slight modification of [Lancaster and Siegel 1996b, Example 2]. One motivation for seeking examples containing central fans (or continuity) is the insight into the central fan question these examples might provide. The cited examples use comparison arguments which require $\kappa>0$; in contrast, our examples illustrate the limiting case of $\kappa=0$. Our solutions are minimal surfaces satisfying contact angle boundary conditions. Such solutions have qualitatively different behavior than solutions of (1a) with $\kappa>0$, and, for example, the a priori height bounds, which play a critical role in the cited examples, do not hold when $\kappa=0$. Our examples are obtained by solving Riemann-Hilbert problems, representing minimal surfaces in terms of Weierstrass $f$ and $g$ representations, and proving that these surfaces behave as claimed in Theorems 3.1, 3.2, 7.1, and 7.2. In principle, our solutions are constructive; computing one of our surfaces would require computing a conformal map and a certain number of complex contour integrals.

\section{Radial limits, central fans and continuity at corners}

Consider $\Omega \subset \mathbb{R}^{2}$ whose boundary has a corner of size $2 \alpha$ at the origin $O=(0,0)$ as described above, and assume the limits in (2) both exist.

Definition. For a function $h$ with domain $\Omega \subset \mathbb{R}^{2}$ and $P \in \partial \Omega$, the radial limit of $h$ at $P$ in the direction $\theta$ is

$$
R h(\theta)=R h(\theta ; P)=\lim _{r \rightarrow 0^{+}} h(P+r \omega(\theta)), \quad \text { where } \omega(\theta)=(\cos (\theta), \sin (\theta)) ;
$$

here $\theta$ is an angle for which $P+r \omega(\theta) \in \Omega$ when $r>0$ is small. (The existence of each limit above follows from [Lancaster and Siegel 1996b, Theorem 1] when $h$ satisfies (1), $\gamma$ is piecewise continuous with $\gamma \in[\epsilon, \pi-\epsilon]$ for some $\epsilon>0$, and $\left|\gamma_{1}+\gamma_{2}-\pi\right|<2 \alpha$.)

2.1. Convex corners. Lancaster and Siegel [1996b, Corollary 4] proved that a solution $h$ of (1) must be continuous at $O$ if $\alpha \leq \frac{\pi}{2}, \quad\left|\gamma_{1}-\gamma_{2}\right| \leq \pi-2 \alpha$, and $\left|\gamma_{1}+\gamma_{2}-\pi\right|<2 \alpha$. The proof follows from lower bounds on the sizes of "fans" of constant radial limits obtained in [Lancaster and Siegel 1996b, Theorem 2]. These bounds implied the "fans" touch or overlap and hence $R h$ is a constant 
function on $[-\alpha, \alpha]$, where $R h( \pm \alpha)$ are defined as the one-sided limits at $O$ of the trace of $h$. These limits are proven to exist as part of the proof of [Theorem 1]; if $h$ is discontinuous at $O$, [Theorem 1] implies $R h$ is not constant. When $\left|\gamma_{1}+\gamma_{2}-\pi\right|>2 \alpha$, we know that no solution of (1) can remain bounded near $O$; see [Concus and Finn 1974b; Concus and Finn 1974a]. When $\left|\gamma_{1}+\gamma_{2}-\pi\right|=2 \alpha$, a variation of Tam's argument [1986] should establish the continuity of a solution at $P$. The Concus-Finn conjecture was recently proven by Lancaster $[\geq 2008]$; hence $h$ is discontinuous at the corner if $\left|\gamma_{1}-\gamma_{2}\right|>\pi-2 \alpha$. Thus, assuming the successful application of Tam's arguments, the continuity or discontinuity of a solution of (1) at a convex corner is completely determined by "local" geometric information, that is, $\gamma_{1}, \gamma_{2}$, and $\alpha$. When $\alpha \leq \pi / 2, h$ is continuous at $O$ if and only if $\left|\gamma_{1}+\gamma_{2}-\pi\right| \leq 2 \alpha$ and $\left|\gamma_{1}-\gamma_{2}\right| \leq \pi-2 \alpha$.

2.2. Nonconvex corners. We now discuss the results of [Lancaster and Siegel 1996b]. The conclusion of [Theorem 1] is that the radial limits of $h$ behave in one of the following ways:

(i) There exist $\alpha_{1}$ and $\alpha_{2}$ such that $-\alpha \leq \alpha_{1}<\alpha_{2} \leq \alpha$ and such that Rh is constant on $\left[-\alpha, \alpha_{1}\right]$ and $\left[\alpha_{2}, \alpha\right]$ and strictly increasing or strictly decreasing on $\left[\alpha_{1}, \alpha_{2}\right]$. Label these case (I) and case (D), respectively.

(ii) There exist $\alpha_{1}, \alpha_{L}, \alpha_{R}, \alpha_{2}$ such that $-\alpha \leq \alpha_{1}<\alpha_{L}<\alpha_{R}<\alpha_{2} \leq \alpha$, where $\alpha_{R}=\alpha_{L}+\pi$ and such that $R h$ is constant on $\left[-\alpha, \alpha_{1}\right],\left[\alpha_{L}, \alpha_{R}\right]$, and $\left[\alpha_{2}, \alpha\right]$ and either strictly increasing on $\left[\alpha_{1}, \alpha_{L}\right]$ and strictly decreasing on $\left[\alpha_{R}, \alpha_{2}\right]$ or strictly decreasing on $\left[\alpha_{1}, \alpha_{L}\right]$ and strictly increasing on $\left[\alpha_{R}, \alpha_{2}\right]$. Label these case (ID) and case (DI), respectively.

We note that the language in (ii) is a little ambiguous since the word "strictly" is not present; an examination of [Parts 2 and 3] of the proof of [Theorem 1] demonstrates that the statement above is correct and the size of the "central fan" $\left[\alpha_{L}, \alpha_{R}\right]$, in directions for which $R h$ is constant, is exactly $\pi$. When $\kappa=\lambda=0$ in (1a), that the size of the central fan is exactly $\pi$ was obtained in [Lancaster 1988, Theorem 2]. The conclusion of [Lancaster and Siegel 1996b, Theorem 2] is that there exist lower bounds on the sizes of the "side fans" $\left[-\alpha, \alpha_{1}\right]$ and $\left[\alpha_{2}, \alpha\right]$ depending on which of the cases (I), (D), (ID), or (DI) holds; these bounds are

- $\alpha_{1}+\alpha \geq \gamma_{2}$ for (D) and (DI);

- $\alpha_{1}+\alpha \geq \pi-\gamma_{2}$ for (I) and (ID);

- $\alpha-\alpha_{2} \geq \gamma_{1}$ for (I) and (DI);

- $\alpha-\alpha_{2} \geq \pi-\gamma_{1}$ for (D) and (ID).

Central fans will be of particular interest to us. [Lancaster and Siegel 1996b, Example 2] is a nonparametric capillary surface, perhaps the first in which a central 
fan can be seen to exist. Shi [2006] assumes the Concus-Finn conjecture holds and proves that if $\kappa>0$ and $\alpha>\pi / 2$ and either $\left|\gamma_{1}-\gamma_{2}\right|>2 \alpha-\pi$ or $\left|\gamma_{1}+\gamma_{2}-\pi\right|>$ $2 \pi-2 \alpha$, then a solution $h$ of (1) must be discontinuous at $O$. Since the ConcusFinn conjecture has been proven, this means that the question of continuity at a nonconvex corner reduces to the situation where the size $2 \alpha$ of the opening angle and the limiting contact angles $\gamma_{1}$ and $\gamma_{2}$ are related by

$$
\left|\gamma_{1}-\gamma_{2}\right| \leq 2 \alpha-\pi \quad \text { and } \quad\left|\gamma_{1}+\gamma_{2}-\pi\right| \leq 2 \pi-2 \alpha \text {. }
$$

When $\left|\gamma_{1}+\gamma_{2}-\pi\right| \leq 2 \pi-2 \alpha$, we claim that the only possibilities are that $h$ is discontinuous at the corner $O$ and (i) holds at $O$ or that $h$ is continuous at $O$. To see this, suppose $h$ is discontinuous at $O$ and either case (ID) or (DI) holds. If (ID) holds, then $\alpha-\alpha_{2} \geq \pi-\gamma_{1}$ and $\alpha_{1}-(-\alpha) \geq \pi-\gamma_{2}$, and (using the fact that central fans are of size $\pi$ ) the sum of the sizes of the three fans is at least $\pi-\gamma_{1}+\pi+\pi-\gamma_{2}$. If (DI) holds, then $\alpha-\alpha_{2} \geq \gamma_{1}$ and $\alpha_{1}-(-\alpha) \geq \gamma_{2}$, and the sum of the sizes of the three fans is at least $\gamma_{1}+\pi+\gamma_{2}$. Now $\left|\gamma_{1}+\gamma_{2}-\pi\right| \leq 2 \pi-2 \alpha$ implies $2 \alpha \leq \pi+\gamma_{1}+\gamma_{2}$ and $2 \alpha \leq 3 \pi-\gamma_{1}-\gamma_{2}$, and these imply that the sum of the sizes of the fans is at least $2 \alpha$; since $R h$ is constant on each fan, $R h$ must be constant, and our assumption that $h$ is discontinuous at $O$ is false. We see therefore that a necessary and sufficient condition for the continuity of $h$ at $O$ is that $\left|\gamma_{1}+\gamma_{2}-\pi\right| \leq 2 \pi-2 \alpha$ and the radial limits of $h$ cannot be monotonic, that is, [Lancaster and Siegel 1996b, Theorem 1(i)] cannot hold. Consider the following:

Definition (symmetry condition). We say that a capillary problem (1) satisfies an "(even) symmetry condition at a point $P \in \partial \Omega$ " if there is a rigid motion $L$ : $\mathbb{R}^{2} \rightarrow \mathbb{R}^{2}$ such that $L(P)=O, L(\Omega)$ is symmetric with respect to the $x$-axis and $\gamma \circ L^{-1}(x,-y)=\gamma \circ L^{-1}(x, y)$ for all $(x, y) \in L(\partial \Omega)$.

[Lancaster and Siegel 1996b, Corollary 2] is the first result which provides sufficient conditions for the existence of a central fan; it implies this: Suppose $\kappa \geq 0$, $\Omega$ has a reentrant (that is, nonconvex) corner at $P \in \partial \Omega$, (1) satisfies the symmetry condition at $P$, and $h$ satisfies (1). Then $h^{*}=h \circ L^{-1}$ is even in y and $R h^{*}(\theta ; O)$ is constant for $\theta \in[-\pi / 2, \pi / 2]$.

Shi and Finn [2004] examined [Lancaster and Siegel 1996b, Example 2] and modified its construction to obtain a $\kappa>0$ capillary surface in a nonsymmetric domain; their solution satisfied the angle requirements of [Lancaster and Siegel 1996b, Corollary 2], that is, (3) with $\gamma_{1}=\gamma_{2}$, but was discontinuous at $O$. The ShiFinn construction made, in a sense they explained, an arbitrarily small perturbation in the domain which left a neighborhood of $O$ unaltered.

We define the central fan question to be the problem of finding necessary and sufficient conditions for excluding [Lancaster and Siegel 1996b, Theorem 1(i)], that is, case (I) and case (D), at a reentrant corner; if cases (I) and (D) are excluded, 
then one of case (ID), case (DI), or $R h$ is constant (and $h$ is continuous at the corner) must hold, and so there exists a central fan of constant radial limits (of size $\pi$ in cases (ID) and (DI) or of size $2 \alpha$ otherwise) at the corner. We consider this question to be the most important open problem in the mathematical theory of capillarity for these reasons:

(i) The (unresolved aspect of the) continuity question - that is, "Is a solution $h$ of (1) continuous or discontinuous at a corner?"- is a special case of the central fan question.

(ii) Any sufficient condition for the existence of central fans will probably be "unstable", as illustrated in [Shi and Finn 2004].

(iii) It seems likely that this question will be very challenging and may require the development of new mathematical techniques or the modification of existing techniques.

(iv) The central fan question has inherent geometric and analytic appeal. The question was first raised by Finn in [1998], and [Shi and Finn 2004] is the first investigation of this question; see also [Finn 1999; Finn 2002].

(v) A solution of this problem offers the possibility of obtaining insight about boundary value problems for general quasilinear elliptic partial differential equations.

\section{Construction of the domains $\Omega$ and main theorems}

We will construct an appropriate "capillary graph" (for example, [Huff and McCuan 2006]) using a Weierstrass representation of minimal surfaces. We assume the domain $\Omega$ is symmetric about the coordinate axes and starshaped as indicated in Figure 1. We assume $\cos (\gamma(x, y))$ is an odd function of $x$ and $y$. In each quadrant, $\partial \Omega$ has a reentrant corner and our principal interest is in the behavior of the solution at these corners. We note that the symmetry condition does not hold at $P$ (nor at $Q, R$, and $S$ ) if $\gamma_{1} \neq \gamma_{2}$; specifically, $h$ will not be symmetric with respect to the line through $P$ that bisects angle $A P B$ in Figure 1 if $\gamma_{1} \neq \gamma_{2}$. We will restrict our attention to $\Omega_{0}$, which is the quarter of the domain in the second quadrant and require the Gauss map of the surface $z=h$ over $\Omega_{0}$ to be injective. This requirement that the Gauss map be injective is critical for our construction using the Weierstrass $(f, g)$-representation of minimal surfaces; however, minimal surfaces with injective Gauss maps over nonconvex domains are in some sense rare and this requirement will "pick out" (depending on $\gamma$ ) the particular domain $\Omega$ in which we will work.

Specifically, to create our domain, we let $\delta \in(0, \pi / 4)$ and, for $B=\left(x_{0}, 0\right)$ with $x_{0} \in(-\cot (\delta),-\tan (\delta))$, consider the domain $\Omega(B) \subset \mathbb{R}^{2}$ illustrated in Figure 1 


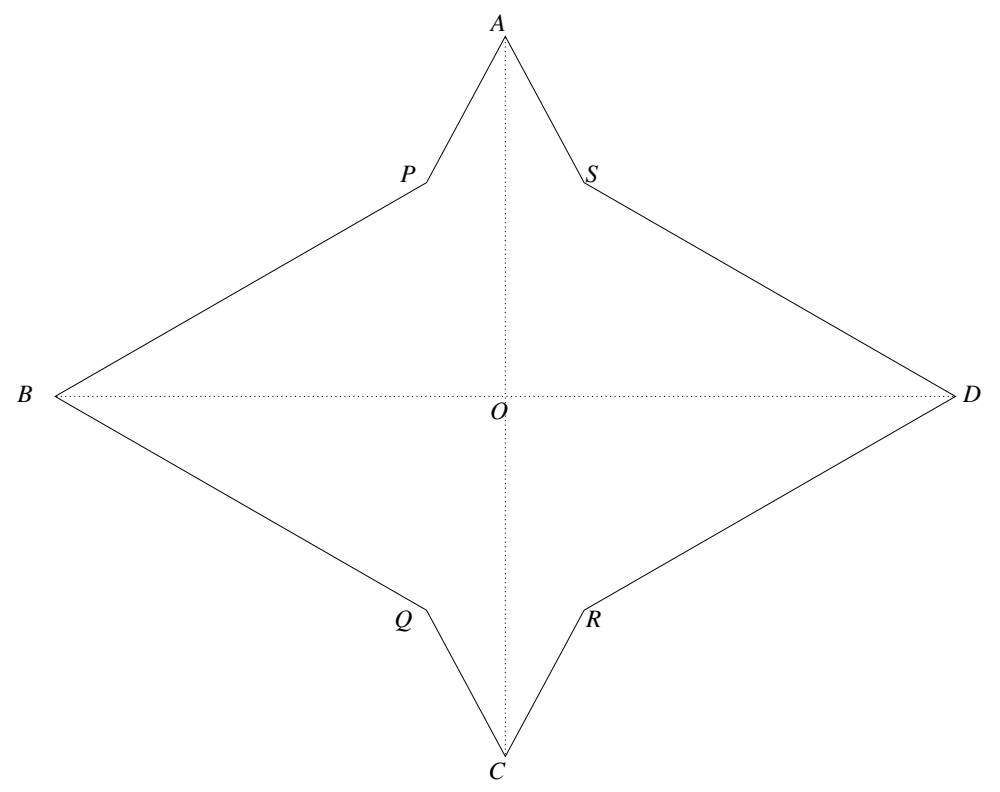

Figure 1. The domain $\Omega$.

whose boundary is a polygon with eight sides that is symmetric with respect to the horizontal and vertical axes and whose two sides in the second quadrant have vertices at $(0,1), P$, and $B$, where $P=P\left(x_{0}\right)$ is the point of intersection of the lines $y=\cot (\delta) x+1$ and $y=\tan (\delta)\left(x-x_{0}\right)$. We have labeled the vertices of $\partial \Omega$, starting at $(0,1)$ and moving in a counterclockwise direction, as $A, P, B, Q, C$, $R, D$, and $S$; we labeled the origin as $O$. The convex angles $O A P$ and $O B P$ have measure $\delta$, and the nonconvex angle $A P B$ has measure $3 \pi / 2-2 \delta$. Let $\Omega_{0}(B)$ be the (open) region whose boundary is the nonconvex quadrilateral $A P B O$.

Consider $\Omega=\Omega(B)$ as the cross section of a vertical cylinder $\Lambda$. We need to specify our contact angle boundary condition on $\Lambda$. Let $\gamma_{1}, \gamma_{2} \in(0, \pi / 2)$ satisfy the condition

$$
\tan (\delta)<\frac{\cos \left(\gamma_{2}\right)}{\cos \left(\gamma_{1}\right)}<\cot (\delta)
$$

and define the function $\gamma: \partial \Omega \rightarrow \mathbb{R}$ by

$$
\gamma(x, y)= \begin{cases}\gamma_{1} & \text { if }(x, y) \in A P \cup C R, \\ \gamma_{2} & \text { if }(x, y) \in P B \cup R D, \\ \pi-\gamma_{2} & \text { if }(x, y) \in B Q \cup D S, \\ \pi-\gamma_{1} & \text { if }(x, y) \in Q C \cup S A .\end{cases}
$$


Consider the capillary problem (1) in the cylinder $\Lambda$ with contact angle boundary data $\gamma$, gravitational constant $\kappa \geq 0$, and Lagrange multiplier $\lambda$. We are interested in the behavior of the solution in zero gravity. In this case, $\kappa=0$ and the divergence theorem together with (1) implies

$$
\lambda|\Omega|=\int_{\Omega} \operatorname{div}(T h) d A=\int_{\partial \Omega} \cos (\gamma) d s .
$$

Since $\cos (\gamma(x, y))$ is an odd function of $x$ and of $y$, we see that $\lambda=0$. This means the solution $h$ will be a minimal surface.

First we will consider pairs $\left(\gamma_{1}, \gamma_{2}\right)$ of contact angles that satisfy the additional restrictions $\gamma_{1} \geq \delta$ and $\gamma_{2} \geq \delta$. It turns out that the simplest case to state, Theorem 3.1 , is the most difficult to prove. In Section 7, we will discuss the situation in which one or both of the assumptions $\gamma_{1} \geq \delta$ and $\gamma_{2} \geq \delta$ is removed. This results in a number of cases, illustrated in Figures 5 and 6 , which affect the geometry of the stereographic projection of the Gauss map; however each case follows rather easily from the proofs of Theorems 3.1 and 3.2.

Theorem 3.1. Let $\delta \in(0, \pi / 4)$ and $\gamma_{1}, \gamma_{2} \in(0, \pi / 2)$ satisfy (4),

$$
\gamma_{1}+\gamma_{2} \geq \pi / 2-2 \delta, \quad \gamma_{1} \geq \delta, \quad \text { and } \quad \gamma_{2} \geq \delta .
$$

There exists a unique $x_{0} \in(-\cot (\delta),-\tan (\delta))$ such that if $B=\left(x_{0}, 0\right), \Omega=\Omega(B)$, and $\Omega_{0}=\Omega_{0}(B)$, then the boundary value problem (1) with $\kappa=\lambda=0$ and $\gamma$ as defined above has a (unique) solution $h \in C^{2}(\Omega) \cap C^{0}(\bar{\Omega})$ which satisfies

(i) $h(0,0)=0$ and

(ii) the Gauss map $\tilde{G}$ of the surface $\Gamma_{0}=\left\{(x, y, h(x, y)):(x, y) \in \Omega_{0}\right\}$ defined by

$$
\tilde{G}(x, y, h(x, y))=\frac{(\nabla h(x, y),-1)}{\sqrt{1+|\nabla h(x, y)|^{2}}} \quad \text { for }(x, y) \in \Omega_{0},
$$

extends to an injective map $\tilde{G} \in C^{0}\left(\overline{\Gamma_{0}}: \mathbf{S}^{2}\right)$.

In addition, if $\gamma_{1}+\gamma_{2}>\pi / 2-2 \delta, \gamma_{1}>\delta$ and $\gamma_{2}>\delta$, then $h \in C^{1}(\bar{\Omega})$.

Theorem 3.2. Let $\delta \in(0, \pi / 4)$ and $\gamma_{1}, \gamma_{2} \in(0, \pi / 2)$ satisfy (4),

$$
\gamma_{1}+\gamma_{2}<\pi / 2-2 \delta, \quad \gamma_{1} \geq \delta, \quad \text { and } \quad \gamma_{2} \geq \delta .
$$

For each $\theta_{1} \in\left[\delta+\gamma_{2}-\pi,-\pi / 2-\delta-\gamma_{1}\right]$, there exists a unique $x_{0}=x_{0}\left(\theta_{1}\right) \in$ $(-\cot (\delta),-\tan (\delta))$ such that if $B=\left(x_{0}, 0\right), \Omega=\Omega(B)$, and $\Omega_{0}=\Omega_{0}(B)$, then the boundary value problem (1) with $\kappa=\lambda=0$ and $\gamma$ as defined above has $a$ (unique) solution $h \in C^{2}(\Omega) \cap C^{0}(\bar{\Omega} \backslash\{P, Q, R, S\})$ which satisfies

(i) $h(0,0)=0$ and 
(ii) the Gauss map $\tilde{G}$ over $\Omega_{0}$, given in (5), extends to an injective map $\tilde{G} \in$ $C^{0}\left(\overline{\Gamma_{0}}: \mathbf{S}^{2}\right)$, where $\Gamma_{0}=\left\{(x, y, h(x, y)):(x, y) \in \Omega_{0}\right\}$.

This solution is discontinuous at $P, Q, R$, and $S$ and

$$
R h(\theta ; P)= \begin{cases}\text { constant } & \text { if } \theta \in\left[\delta-\pi, \delta+\gamma_{2}-\pi\right], \\ \text { strictly decreasing } & \text { if } \theta \in\left[\delta+\gamma_{2}-\pi, \theta_{1}\right], \\ \text { constant } & \text { if } \theta \in\left[\theta_{1}, \theta_{1}+\pi\right], \\ \text { strictly increasing } & \text { if } \theta \in\left[\theta_{1}+\pi, \pi / 2-\delta-\gamma_{1}\right], \\ \text { constant } & \text { if } \theta \in\left[\pi / 2-\delta-\gamma_{1}, \pi / 2-\delta\right] .\end{cases}
$$

$R h(\theta ; Q), R h(\theta ; R)$, and $R h(\theta ; S)$ behave in appropriately similar manners. In particular, $\theta_{1}$ determines the position of the central fan and, through the determination of the point $B$, the geometry of the domain.

\section{Proof preliminaries}

We are interested in the stereographic projection $E$ of the image of the Gauss map of our solution over $\overline{\Omega_{0}(B)}$ for an appropriate $B$. In this section, we assume $\gamma_{i} \geq \delta$ for $i=1,2$. To determine $E$, let $B_{1}=\{w \in \mathbb{C}:|w|<1\}, Q_{1}=\left\{w \in B_{1}: \operatorname{Re}(w)<\right.$ $0, \operatorname{Im}(w)>0\}$ and set

$$
E_{0}=\left\{w \in Q_{1}:\left|w-w_{1}\right|>\tan \left(\gamma_{1}\right),\left|w-w_{2}\right|>\tan \left(\gamma_{2}\right)\right\},
$$

where $w_{1}=u_{1}+i v_{1}=-\cos (\delta) \sec \left(\gamma_{1}\right)+i \sin (\delta) \sec \left(\gamma_{1}\right)$ and $w_{2}=u_{2}+i v_{2}=$ $-\sin (\delta) \sec \left(\gamma_{2}\right)+i \cos (\delta) \sec \left(\gamma_{2}\right)$. The constant contact angle condition on the vertical plane $\overline{A P} \times \mathbb{R}$ corresponds to requiring that the Gauss map lie on the circle that is the intersection of $S^{2}$ and a cone with opening angle $\gamma_{1}$. The stereographic projection of this circle is the circle $C_{1}=\left\{w:\left|w-w_{1}\right|=\tan \left(\gamma_{1}\right)\right\}$ centered at $w_{1}$. In the same way, we see that the constant contact angle condition on the vertical plane $\overline{P B} \times \mathbb{R}$ corresponds to requiring that the Gauss map lie on the circle that is the intersection of $S^{2}$ and a cone with opening angle $\gamma_{2}$. The stereographic projection of this second circle is $C_{2}=\left\{w:\left|w-w_{2}\right|=\tan \left(\gamma_{2}\right)\right\}$. Then $E=\overline{E_{0}}$.

Let us examine the set $E$, which is a simply connected subset of the closed unit disk. The boundary of $E$ consists of portions of the circles $C_{1}$ and $C_{2}$ which are orthogonal to the unit circle $\partial B_{1}$, the positive imaginary axis, the negative real axis and (possibly) the unit quarter-circle $\left\{w \in \partial B_{1}: \operatorname{Re}(w) \leq 0, \operatorname{Im}(w) \geq 0\right\}$. If the circles $C_{1}$ and $C_{2}$ intersect in $|w| \leq 1$, then let $w_{0}=u_{0}+i v_{0}$ be this point of intersection; (4) implies $w_{0} \in Q_{1}$, and by reflecting about $\partial B_{1}$, we see that

$$
\left(v_{2}-v_{1}\right) v_{0}=\left(u_{1}-u_{2}\right) u_{0} \text {. }
$$


Note that $C_{1}$ lies entirely in the open set $\operatorname{Re}(w)<0$ if $\gamma_{1}<\pi / 2-\delta$, and $C_{2}$ lies entirely in the open set $\operatorname{Im}(w)>0$ if $\gamma_{2}<\pi / 2-\delta$. Let us write

$$
\begin{array}{ll}
\sigma_{1}=\partial E \cap C_{1}, & \sigma_{3}=\{w \in \partial E: \operatorname{Im}(w)=0\}, \\
\sigma_{2}=\partial E \cap C_{2}, & \sigma_{4}=\{w \in \partial E: \operatorname{Re}(w)=0\}, \\
\sigma_{5}=\{w \in \partial E:|w|=1\} .
\end{array}
$$

We will set $t_{0}=0$. We denote the remaining corners of $\partial E$ as $t_{1}, \ldots, t_{L}$ for $3 \leq$ $L \leq 4$. The $t_{i}$ have these properties:

(i) $t_{1}, t_{2} \in \sigma_{1}$ and $\operatorname{Im}\left(t_{2}\right)=0$; that is, $t_{2}$ is the intersection of $C_{1}$ with the negative real axis, and $t_{1}$ is the intersection of $C_{1}$ with either $C_{2}$ (see (iii)) or $\partial B_{1}$ (see (iv)).

(ii) $t_{3} \in \sigma_{2}$ and $\operatorname{Re}\left(t_{3}\right)=0$; that is, $t_{3}$ is the intersection of $C_{2}$ with the positive imaginary axis.

(iii) $\gamma_{1}+\gamma_{2} \geq \pi / 2-2 \delta$ implies $L=3$ and $t_{1} \in \sigma_{2}$.

(iv) $\gamma_{1}+\gamma_{2}<\pi / 2-2 \delta$ implies $L=4, t_{4} \in \sigma_{2}$ and $t_{1}, t_{4} \in \partial B_{1}$.

When condition (iii) holds, Figure 2 illustrates the shape of $E$, while Figure 3 illustrates the shape of $E$ when condition (iv) holds. An example of a listing of the corners in counterclockwise order is $t_{1}, t_{2}, t_{0}, t_{3}$, and, if $L=4, t_{4}$. Notice that when condition (iv) holds, $t_{1}=\exp \left(\left(\pi-\delta-\gamma_{1}\right) i\right)$ and $t_{4}=\exp \left(\left(\pi / 2+\delta+\gamma_{2}\right) i\right)$.

For the Weierstrass representation, let us define $g: E \rightarrow E$ by $g(w)=w$. We wish to find $f \in C^{0}\left(E \backslash\left\{t_{1}, \ldots, t_{L}\right\}\right)$, with at worst integrable singularities at $t_{1}, \ldots, t_{L}$, which is analytic in $E_{0}$. We define $X \in C^{0}\left(E: \mathbb{R}^{3}\right) \cap C^{2}\left(E_{0}: \mathbb{R}^{3}\right)$ with

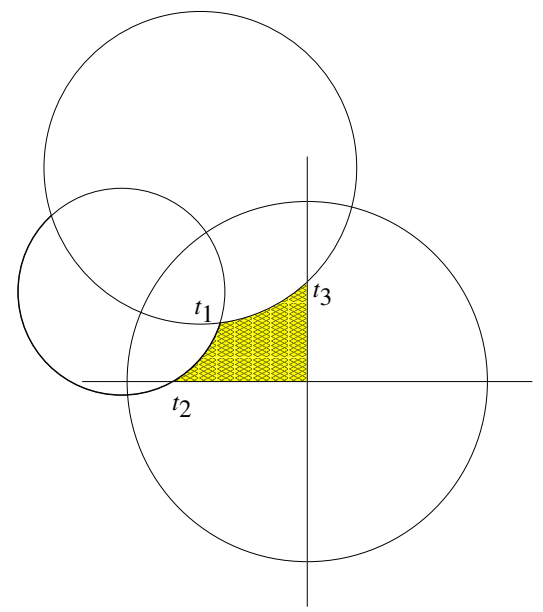

Figure 2. $\gamma_{1} \geq \delta, \gamma_{2} \geq \delta, \gamma_{1}+\gamma_{2} \geq \pi / 2-2 \delta$. 


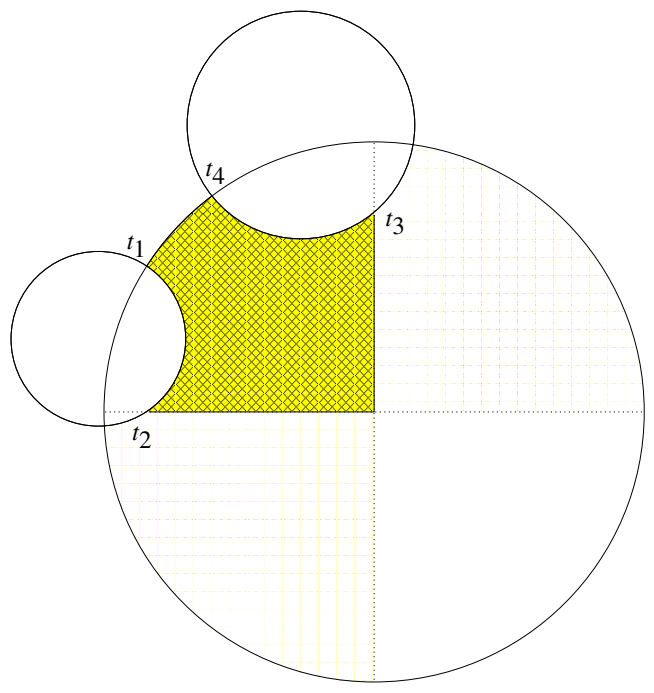

Figure 3. $\gamma_{1} \geq \delta, \gamma_{2} \geq \delta, \gamma_{1}+\gamma_{2}<\pi / 2-2 \delta$.

$$
X(u+i v)=(x(u, v), y(u, v), z(u, v)) .
$$

and $K(u+i v)=(x(u, v), y(u, v))$ for $u+i v \in E$, such that $X(0)=(0,0,0)$ and (a) the analytic functions $(f, g)$ form the Weierstrass representation of $X$ [Osserman 1986]; (b) $K$ is a homeomorphism between $\sigma_{1}$ and $A P, \sigma_{2}$ and $P B, \sigma_{3}$ and $O A$, and $\sigma_{4}$ and $B O$ (where, for example, $O A$ means the line segment between $O$ and $A)$; and (c)

$K$ is constant on each component of $\sigma_{5}$.

Here we say $f$ has an integrable singularity at $t_{n}$ if and only if $|f(w)| \leq C\left|w-t_{n}\right|^{s}$ for $-1<s<0$ and $C \geq 0$ for $w$ near $t_{n}$.

We will now formulate the Riemann-Hilbert problem which we will solve by temporarily assuming the existence of a suitable function $f$.

The boundary requirements (b) imply

$$
\begin{array}{ll}
y(u, v)=\cot (\delta) x(u, v)+1 & \text { for } u+i v \in \sigma_{1}, \\
y(u, v)=\tan (\delta)\left(x(u, v)-x_{0}\right) & \text { for } u+i v \in \sigma_{2}, \\
x(u, v)=0 \quad \text { and } \quad 0 \leq y(u, v) \leq 1 & \text { for } u+i v \in \sigma_{3}, \\
y(u, v)=0 \quad \text { and } \quad x_{0} \leq x(u, v) \leq 0 & \text { for } u+i v \in \sigma_{4},
\end{array}
$$

where $x_{0} \in(-\cot (\delta),-\tan (\delta))$ remains to be determined. Decompose $f(u+i v)=$ 
$f_{1}(u, v)+i f_{2}(u, v)$, where $f_{1}$ and $f_{2}$ are real-valued. Now (a) implies

$$
x_{w}=f(w)\left(1-w^{2}\right) / 2, \quad y_{w}=i f(w)\left(1+w^{2}\right) / 2, \quad z_{w}=w f(w)
$$

for $w \in E$; see for example [Osserman 1986]. Since $d / d w=(\partial / \partial u-i \partial / \partial v) / 2$, the equations above yield

$$
\begin{array}{ll}
x_{u}(u, v)=\operatorname{Re}\left(f(w)\left(1-w^{2}\right)\right), & x_{v}(u, v)=-\operatorname{Im}\left(f(w)\left(1-w^{2}\right)\right), \\
y_{u}(u, v)=\operatorname{Re}\left(i f(w)\left(1+w^{2}\right)\right), & y_{v}(u, v)=-\operatorname{Im}\left(i f(w)\left(1+w^{2}\right)\right), \\
z_{u}(u, v)=\operatorname{Re}(2 w f(w)), & z_{v}(u, v)=-\operatorname{Im}(2 w f(w)),
\end{array}
$$

where we use the notation $w=u+i v$.

If we parametrize $\sigma_{k}$ by $\sigma_{k}=\left\{w_{k}(t)=u_{k}(t)+i v_{k}(t): t \in I_{k}\right\}$, with $I_{k}$ a closed interval in $\mathbb{R}$ for $k=1, \ldots, 5$, then by differentiating with respect to $t$ (and denoting $d / d t$ by a prime), we find

(8a) implies $y_{u} u_{1}^{\prime}+y_{v} v_{1}^{\prime}=\cot (\delta)\left(x_{u} u_{1}^{\prime}+x_{v} v_{1}^{\prime}\right)$;

(8b) implies $y_{u} u_{2}^{\prime}+y_{v} v_{2}^{\prime}=\tan (\delta)\left(x_{u} u_{2}^{\prime}+x_{v} v_{2}^{\prime}\right)$;

(8c) implies $x_{u}\left(u_{3}(t), 0\right)=0$;

(8d) implies $y_{v}\left(0, v_{4}(t)\right)=0$; and

(7) implies $x_{u} u_{5}^{\prime}+x_{v} v_{5}^{\prime}=0$ and $y_{u} u_{5}^{\prime}+y_{v} v_{5}^{\prime}=0$.

For $\sigma_{1}$, we have $\left(u_{1}(t)-u_{1}\right)^{2}+\left(v_{1}(t)-v_{1}\right)^{2}=r_{1}^{2}$, where $r_{1}=\tan \left(\gamma_{1}\right)$, which implies

$$
\frac{u_{1}^{\prime}(t)}{v_{1}^{\prime}(t)}=-\frac{v_{1}(t)-v_{1}}{u_{1}(t)-u_{1}}=-\frac{\operatorname{Im}\left(w_{1}(t)-w_{1}\right)}{\operatorname{Re}\left(w_{1}(t)-w_{1}\right)} .
$$

For $\sigma_{2}$, we have $\left(u_{2}(t)-u_{2}\right)^{2}+\left(v_{2}(t)-v_{2}\right)^{2}=r_{2}^{2}$, where $r_{2}=\tan \left(\gamma_{2}\right)$, which implies

$$
\frac{u_{2}^{\prime}(t)}{v_{2}^{\prime}(t)}=-\frac{v_{2}(t)-v_{2}}{u_{2}(t)-u_{2}}=-\frac{\operatorname{Im}\left(w_{2}(t)-w_{2}\right)}{\operatorname{Re}\left(w_{2}(t)-w_{2}\right)} .
$$

Recall that $\cot (\delta)=-\operatorname{Re}\left(w_{1}\right) / \operatorname{Im}\left(w_{1}\right)=-u_{1} / v_{1}$ and $\tan (\delta)=-\operatorname{Re}\left(w_{2}\right) / \operatorname{Im}\left(w_{2}\right)=$ $-u_{2} / v_{2}$.

If we use this information, we obtain

$$
\operatorname{Re}\left(\left(a_{k}(u, v)+i b_{k}(u, v)\right) f(u+i v)\right)=0,
$$

when $(u, v) \in \sigma_{k}$ for $k=1, \ldots, 5$, where

$$
\begin{array}{ll}
a_{1}(u, v)+i b_{1}(u, v)=i e^{i \delta}\left(w-w_{1}\right)\left(e^{-2 \delta i}-w^{2}\right) & \text { if } w=u+i v \in \sigma_{1}, \\
a_{2}(u, v)+i b_{2}(u, v)=e^{-i \delta}\left(w-w_{2}\right)\left(e^{2 \delta i}+w^{2}\right) & \text { if } w=u+i v \in \sigma_{2}, \\
a_{3}(u, v)+i b_{3}(u, v)=-1 & \text { if } w=u+i v \in \sigma_{3}, \\
a_{4}(u, v)+i b_{4}(u, v)=-1 & \text { if } w=u+i v \in \sigma_{4}, \\
a_{5}(u, v)+i b_{5}(u, v)=(u+i v)^{2} & \text { if } w=u+i v \in \sigma_{5} .
\end{array}
$$


We now define $a, b: \partial E \rightarrow \mathbb{R}$ by $a(u+i v)=a_{k}(u, v)$ and $b(u+i v)=b_{k}(u, v)$ if $u+i v \in \sigma_{k}$ for $k \in\{1, \ldots, 5\}$ and define $G: \partial E \rightarrow \mathbb{C}$ by $G(w)=a(w)+i b(w)$. It is possible that $\sigma_{5}=\varnothing$, in which case $a_{5}(u, v)+i b_{5}(u, v)$ has no impact on $G$.

Assuming the existence of $f$, we were led to (9). Now we can formulate the problem of finding $X$ with $g=$ id. We wish to find a function $f \in C^{0}(E \backslash$ $\left.\left\{t_{1}, \ldots, t_{L}\right\}\right)$ that is analytic in $E_{0}$ and satisfies

$$
\operatorname{Re}(G(w) f(w))=0 \quad \text { for } w \in \partial E \backslash\left\{t_{1}, \ldots, t_{L}\right\} .
$$

This is a Hilbert problem with piecewise discontinuous Hölder coefficients $G$ in the notation of [Monakhov 1983, Chapter 1, Section 4]. To use the results in [Monakhov 1983], we need to compute the index of $G$ in an appropriate function class $O(m)=O\left(t_{n_{1}}, \ldots, t_{n_{m}}\right)$ for some $m \in\{0, \ldots, L\}$. Define $G_{1}: \partial E \rightarrow \mathbb{C}$ by

$$
G_{1}(w)=-\frac{\overline{G(w)}}{G(w)} .
$$

We have $G_{1}(w)=-1$ for $w \in \sigma_{3} \cup \sigma_{4}, G_{1}$ is continuous at 0 , and $G_{1}(w)=$ $-(\bar{w} /|w|)^{4}=-\bar{w}^{4}$ for $w \in \sigma_{5}$. Set $\omega=e^{i \delta}$. Notice also that

$$
\begin{array}{ll}
G_{1}(w)=|G(w)|^{-2}\left(\overline{\omega\left(w-w_{1}\right)\left(\bar{\omega}^{2}-w^{2}\right)}\right)^{2} & \text { for } w \in \sigma_{1}, \\
G_{1}(w)=-|G(w)|^{-2} e^{2 \delta i}\left(\overline{\left(w-w_{2}\right)\left(w^{2}+\omega^{2}\right)}\right)^{2} & \text { for } w \in \sigma_{2} .
\end{array}
$$

For two corners $t_{n}$ and $t_{n+1}$ with $n \geq 1$ on $\partial E$, let $\left[t_{n}, t_{n+1}\right]$ denote the arc of $\partial E$ between $t_{n}$ and $t_{n+1}$ with $t_{L+1}$ equalling $t_{1}$; for example, $\left[t_{1}, t_{2}\right]=\sigma_{1}$ and $\left[t_{2}, t_{3}\right]=\sigma_{3} \cup \sigma_{4}$. For $n \in\{1, \ldots, L\}$, let

$$
\theta_{n}=\frac{1}{2 \pi}\left(\arg \left(G_{1}\left(t_{n}-0\right)\right)-\arg \left(G_{1}\left(t_{n}+0\right)\right)\right),
$$

where $\arg \left(G_{1}\left(t_{n}-0\right)\right)$ means the limit at $t_{n}$ of the argument of $G_{1}$ along the arc $\left[t_{n-1}, t_{n}\right]$ and $\arg \left(G_{1}\left(t_{n}+0\right)\right)$ means the limit at $t_{n}$ of the argument of $G_{1}$ along the $\operatorname{arc}\left[t_{n}, t_{n+1}\right]$. The argument is taken to be continuous along each arc $\left[t_{n}, t_{n+1}\right]$.

To understand the monotonicity behavior of arg $G_{1}$ on the different arcs, and since $G_{1}$ is a product on $\sigma_{1}$ and $\sigma_{2}$, we consider $w \in \overline{Q_{1}} \backslash\{-\bar{\omega}, i \omega\}$ and define

$$
\lambda_{1}(w)=\arg \left(\bar{\omega}^{2}-w^{2}\right) \quad \text { and } \quad \lambda_{2}(w)=\arg \left(\omega^{2}+w^{2}\right)=\arg \left(w^{2}-\left(-\omega^{2}\right)\right) ;
$$

note that $\lambda_{1}(w) \in(-\pi / 2-2 \delta, \pi / 2-2 \delta)$ and $\lambda_{2}(w) \in(-\pi / 2+2 \delta, \pi / 2+2 \delta)$.

Notice that if $w \in \sigma_{3}$, then $\lambda_{1}(w) \in[-\pi / 2-\delta,-2 \delta)$ and if $w \in \sigma_{4}$, then $\lambda_{2}(w) \in$ $(2 \delta, \pi / 2+\delta]$. Let us factor $\bar{\omega}^{2}-w^{2}$ and $\omega^{2}+w^{2}$ and analyze these factors. Define $\alpha_{1}(w)=\arg (w-\bar{\omega}) \in[3 \pi / 4-\delta / 2, \pi-\delta / 2], \alpha_{2}(w)=\arg (w+i \omega) \in[\pi / 2+$ $\delta / 2,3 \pi / 4+\delta / 2], \beta_{1}(w)=\arg (w+\bar{\omega})=\arg (w-(-\bar{\omega})) \in(-\pi / 2-\delta, \pi / 2-\delta)$, and $\beta_{2}(w)=\arg (w-i \omega) \in(\delta-\pi, \delta)$. Then

$$
\lambda_{1}(w)=\alpha_{1}(w)+\beta_{1}(w)-\pi \quad \text { and } \quad \lambda_{2}(w)=\alpha_{2}(w)+\beta_{2}(w) .
$$


Some simple calculations show that if $w_{(1)}(\tau)=w_{1}+r_{1} e^{i \tau}$ parametrizes the circular $\operatorname{arc} C_{1} \cap \overline{B_{1}}$, then

$$
\frac{d}{d \tau}\left(\alpha_{1}\left(w_{(1)}(\tau)\right)\right)<0, \quad \frac{d}{d \tau}\left(\beta_{1}\left(w_{(1)}(\tau)\right)\right)>0, \quad \frac{d}{d \tau}\left(\left(\alpha_{1}+\beta_{1}\right)\left(w_{(1)}(\tau)\right)\right)>0,
$$

while if $w_{(2)}(\tau)=w_{2}+r_{2} e^{i \tau}$ parametrizes the circular arc $C_{2} \cap \overline{B_{1}}$, then

$$
\frac{d}{d \tau}\left(\alpha_{2}\left(w_{(2)}(\tau)\right)\right)<0, \quad \frac{d}{d \tau}\left(\beta_{2}\left(w_{(2)}(\tau)\right)\right)>0, \quad \frac{d}{d \tau}\left(\left(\alpha_{2}+\beta_{2}\right)\left(w_{(2)}(\tau)\right)\right)>0 .
$$

Hence $\lambda_{1}(w)$ is strictly increasing as $w \in \sigma_{1}$ moves in a clockwise direction with respect to $E$ (that is, counterclockwise on $C_{1}$ ) and $\lambda_{2}(w)$ is strictly increasing as $w \in \sigma_{2}$ moves in a clockwise direction with respect to $E$ (that is, counterclockwise on $\left.C_{2}\right)$. Similarly, we see that $\lambda_{1}(u)$ is strictly increasing for $u \in[-1,0]$ and $\lambda_{2}(i v)$ is strictly increasing for $v \in[0,1]$.

\section{Proof of Theorem 3.1}

Suppose first that $\gamma_{1}+\gamma_{2} \geq \pi / 2-2 \delta$, so that $L=3$. Then

$$
\begin{aligned}
& t_{1}=w_{2}+r_{2} e^{i \tau_{2 A}} \text { for some } \tau_{2 A} \in\left[\gamma_{2}+\delta-\pi, 0\right), \\
& t_{3}=w_{2}+r_{2} e^{i \tau_{2 B}} \text { for some } \tau_{2 B} \in(\delta-\pi / 2,0], \text { with } \tau_{2 A}<\tau_{2 B}, \\
& t_{1}=w_{1}+r_{1} e^{i \tau_{1 A}} \text { for some } \tau_{1 A} \in\left(-\pi / 2, \pi / 2-\delta-\gamma_{1}\right], \\
& t_{2}=w_{1}+r_{1} e^{i \tau_{1 B}} \text { for some } \tau_{1 B} \in[-\pi / 2,-\delta), \text { with } \tau_{1 A}>\tau_{1 B} .
\end{aligned}
$$

We observe that

$$
\begin{aligned}
& \alpha_{2}\left(t_{3}\right)=\arg \left(t_{3}+i \omega\right)<\pi / 2+\delta, \quad \beta_{2}\left(t_{3}\right)=\arg \left(t_{3}-i \omega\right)<\delta, \\
& \alpha_{1}\left(t_{2}\right)=\arg \left(t_{2}-\bar{\omega}\right)>\pi-\delta, \quad \beta_{1}\left(t_{2}\right)=\arg \left(t_{2}+\bar{\omega}\right)>-\pi / 2-\delta,
\end{aligned}
$$

$\tau_{1 A}-\tau_{2 A}>0$ and (4) implies $\tau_{2 A}<0$ and $\tau_{1 A}>-\pi / 2$. For the sake of definiteness, we set

$$
\begin{aligned}
& \arg \left(G_{1}\left(t_{1}-0\right)\right)=\pi+2 \delta-2\left(\tau_{2 A}+\lambda_{2}\left(t_{1}\right)\right), \\
& \arg \left(G_{1}\left(t_{1}+0\right)\right)=-2\left(\delta+\tau_{1 A}+\lambda_{1}\left(t_{1}\right)\right), \\
& \arg \left(G_{1}\left(t_{2}-0\right)\right)=-2\left(\delta+\tau_{1 B}+\lambda_{1}\left(t_{2}\right)\right), \\
& \arg \left(G_{1}\left(t_{2}+0\right)\right)=\arg \left(G_{1}\left(t_{3}-0\right)\right)=\pi, \\
& \arg \left(G_{1}\left(t_{3}+0\right)\right)=\pi+2 \delta-2\left(\tau_{2 B}+\lambda_{2}\left(t_{3}\right)\right) .
\end{aligned}
$$

Notice that

$$
\begin{aligned}
& \theta_{1}=1 / 2+\left(2 \delta+\tau_{1 A}-\tau_{2 A}+\lambda_{1}\left(t_{1}\right)-\lambda_{2}\left(t_{1}\right)\right) / \pi, \\
& \theta_{2}=-1 / 2-\left(\delta+\lambda_{1}\left(t_{2}\right)+\tau_{1 B}\right) / \pi \in(-1 / 2+2 \delta / \pi, 1 / 2], \\
& \theta_{3}=\left(\lambda_{2}\left(t_{3}\right)+\tau_{2 B}-\delta\right) / \pi \in(-1 / 2+2 \delta / \pi, 1 / 2] .
\end{aligned}
$$


Also

$$
\begin{aligned}
& \arg \left(G_{1}\left(t_{2}-0\right)\right)-\arg \left(G_{1}\left(t_{1}+0\right)\right)=2\left(\tau_{1 A}-\tau_{1 B}\right)+2\left(\lambda_{1}\left(t_{1}\right)-\lambda_{1}\left(t_{2}\right)\right)>0, \\
& \arg \left(G_{1}\left(t_{1}-0\right)\right)-\arg \left(G_{1}\left(t_{3}+0\right)\right)=2\left(\tau_{2 B}-\tau_{2 A}\right)+2\left(\lambda_{2}\left(t_{3}\right)-\lambda_{2}\left(t_{1}\right)\right)>0 ;
\end{aligned}
$$

hence $\theta_{1}+\theta_{2}+\theta_{3}>0$. Therefore, $\theta_{1}>1-4 \delta / \pi>0$.

Now $\tau_{1 A}-\tau_{2 A}$ equals the angle formed by the triangle with vertices $w_{1}, t_{1}$, and $w_{2}$ at the vertex $t_{1}$; notice that $0<\tau_{1 A}-\tau_{2 A} \leq \pi$. Also, $\beta_{1}\left(t_{1}\right)-\beta_{2}\left(t_{1}\right)$ equals the angle at $t_{1}$ formed by the line segments $\left[t_{1},-\bar{\omega}\right]$ and $\left[t_{1}, i \omega\right]$ from $t_{1}$ to $-\bar{\omega}$ and $i \omega$, respectively, where the angle begins in the direction $\left[t_{1}, i \omega\right]$ and moves counterclockwise to $\left[t_{1},-\bar{\omega}\right]$. Notice that $\tau_{1 A}-\tau_{2 A}<\beta_{1}\left(t_{1}\right)-\beta_{2}\left(t_{1}\right)$ and $\pi / 4-\delta<\beta_{1}\left(t_{1}\right)-\beta_{2}\left(t_{1}\right) \leq 3 \pi / 4+\delta$. Further, $\alpha_{1}\left(t_{1}\right)-\alpha_{2}\left(t_{1}\right)$ equals the (acute) angle formed by the triangle with vertices $-i \omega, t_{1}$, and $\bar{\omega}$ at the vertex $t_{1}$; notice that $\pi / 4-\delta \leq \alpha_{1}\left(t_{1}\right)-\alpha_{2}\left(t_{1}\right)<\pi / 2-2 \delta$. In agreement with the conclusion of the previous paragraph, we see that

$$
\theta_{1}>1 / 2+(2 \delta+0+[(\pi / 4-\delta)+(\pi / 4-\delta)-\pi]) / \pi=0,
$$

where the sum in the square brackets is a lower bound for $\lambda_{1}\left(t_{1}\right)-\lambda_{2}\left(t_{1}\right)$. In fact, as $\gamma_{1}$ and $\gamma_{2}$ converge to $\pi / 2$ from below, $\theta_{2}$ and $\theta_{3}$ each converge to $-1 / 2+2 \delta / \pi$, and $\theta_{1}$ converges to $1-4 \delta / \pi$.

If we set $z_{1}=\exp \left(i\left(\pi-\delta-\gamma_{1}\right)\right)$ and $z_{2}=\exp \left(i\left(\pi / 2+\delta+\gamma_{2}\right)\right)$, so that $z_{1} \in$ $C_{1} \cap \partial B_{1}$ and $z_{2} \in C_{2} \cap \partial B_{1}$, then the monotonicity of $\lambda_{2}$ on $C_{2} \cap B_{1}$ implies

$$
\arg \left(G_{1}\left(t_{1}-0\right)\right) \leq \pi+2 \delta-2\left[\tau_{2 A}+\lambda_{2}\left(z_{2}\right)\right]=2 \pi-2 \tau_{2 A}-2 \delta-2 \gamma_{2},
$$

and the monotonicity of $\lambda_{1}$ on $C_{1} \cap B_{1}$ implies

$$
\arg \left(G_{1}\left(t_{1}+0\right)\right) \geq-2 \delta-2\left[\tau_{1 A}+\lambda_{1}\left(z_{1}\right)\right]=-\pi-2 \tau_{1 A}+2 \delta+2 \gamma_{1} .
$$

Recalling that $\gamma_{1}+\gamma_{2} \geq \pi / 2-2 \delta$, we obtain

$$
0<\theta_{1} \leq 1+\left(\tau_{1 A}-\tau_{2 A}\right) / \pi \leq 2
$$

and $\theta_{1}=2$ if and only if $\gamma_{1}+\gamma_{2}=\pi / 2-2 \delta$.

Set $v_{1}=\left[\theta_{1}\right], \quad v_{2}=0, \quad v_{3}=0, \alpha_{1}=\theta_{1}-v_{1}, \alpha_{2}=\theta_{2}$, and $\alpha_{3}=\theta_{3}$, where $\left[\theta_{1}\right]$ denotes the greatest integer less than or equal to $\theta_{1}$. Notice that $v_{1}+v_{2}+v_{3} \in$ $\{0,1,2\}$. Let $\psi$ be the conformal map from $E$ onto the unit disk $B_{1}$ that maps $t_{1}$ to $1, t_{2}$ to $i$, and $t_{3}$ to $-i$. Define $G^{*}: \partial B_{1} \rightarrow \mathbb{C}$ by

$$
G^{*}(\zeta)=G\left(\psi^{-1}(\zeta)\right) \text { for } \zeta \in \partial B_{1} .
$$

Our Hilbert problem (10) is then equivalent to finding $f^{*} \in C^{0}\left(\overline{B_{1}} \backslash\{1, \pm i\}\right)$ which is analytic in $B_{1}$, has (at worst) integrable singularities at $1, i,-i$, and satisfies

$$
\operatorname{Re}\left(G^{*}(\zeta) f^{*}(\zeta)\right)=0 \quad \text { for } \zeta \in \partial B_{1} \backslash\{1, \pm i\}
$$


We now use results from [Monakhov 1983, Chapter 1, Section 4]. Using [2. ${ }^{\circ}(\mathrm{a})$, p. 49-52] when $v_{1}=0$ after setting $\kappa=0,\left[2 .^{\circ}(\mathrm{b})\right.$, p. 52-53] when $v_{1}=1$ after setting $\kappa=0$, and [2. $\left.{ }^{\circ}(\mathrm{a}), \mathrm{p} .49-52\right]$ when $\nu_{1}=2$ after setting $\kappa=1$, we obtain a solution $f=c f_{0}$ of (10), where $f_{0}$ has the form

$$
f_{0}(w)=(\psi(w)-1)^{\theta_{1}}(\psi(w)-i)^{\alpha_{2}}(\psi(w)+i)^{\alpha_{3}} e^{\Gamma_{0}(\psi(w))}
$$

and $e^{\Gamma_{0}(\zeta)}$ is a continuous, nonvanishing function on $\overline{B_{1}}$. Assuming $c \neq 0, f$ does not vanish on $E \backslash\left\{t_{1}, t_{2}, t_{3}\right\}$. Notice that $\operatorname{Re}\left(f_{0}(w)\right)=0$ if $w \in\left(\sigma_{3} \cup \sigma_{4}\right) \backslash\left\{t_{2}, t_{3}\right\}$, and so $f_{0}(0)=i b_{0}$ for some $b_{0} \neq 0$. Now $\operatorname{Im}\left(f_{0}(w)\right) \neq 0$, and so $\operatorname{Im}\left(b_{0} f_{0}(w)\right)>0$ for $w \in\left(\sigma_{3} \cup \sigma_{4}\right) \backslash\left\{t_{2}, t_{3}\right\}$. We set

$$
-1 / c=\int_{t_{2}}^{0}\left(1+u^{2}\right) i f_{0}(u) d u
$$

and observe that $c b_{0}>0$ since $t_{2}<0$. It follows then that $f(0)=i b_{0} c, \operatorname{Im}(f(w))>$ 0 for $w \in\left(\sigma_{3} \cup \sigma_{4}\right) \backslash\left\{t_{2}, t_{3}\right\}$, and

$$
\int_{0}^{t_{2}}\left(1+u^{2}\right) i f(u) d u=1 .
$$

Let $X \in C^{0}\left(E: \mathbb{R}^{3}\right)$ be the minimal surface whose Weierstrass $(f, g)$ representation is given by $g(w)=w$, where $f$ is defined above, and which satisfies $X(0)=$ $(0,0,0)$. Recall $(6)$ and notice that $\left(x\left(t_{2}, 0\right), y\left(t_{2}, 0\right)\right)=(0,1)$ and $K$ maps $\sigma_{3}$ onto the line segment joining $(0,0)$ and $(0,1)$. Also $x\left(0, t_{3}\right)<0$ and $K$ maps $\sigma_{4}$ onto the line segment joining $(0,0)$ and $B=\left(x\left(0, t_{3}\right), 0\right)$. With $w=u+i v \in E$, let

$$
\vec{N}(w)=\frac{X_{u}(w) \times X_{v}(w)}{\left|X_{u}(w) \times X_{v}(w)\right|}=\left(\frac{2 \operatorname{Re}(w)}{|w|^{2}+1}, \frac{2 \operatorname{Im}(w)}{|w|^{2}+1}, \frac{|w|^{2}-1}{|w|^{2}+1}\right)
$$

denote the Gauss map $\vec{N} \in C^{0}\left(E: S^{2}\right)$ of $X(E)$; here $\vec{N}(w)$ is the downward unit normal to $X(E)$ at $X(w)$.

From the above, setting $h_{2}(w)=(\psi(w)-1)^{\alpha_{1}}(\psi(w)+i)^{\alpha_{3}} e^{\Gamma_{0}(\psi(w))}$, and defining $h_{3}(w)$ similarly, we have

$$
f(w)=(\psi(w)-i)^{\alpha_{2}} h_{2}(w)=(\psi(w)+i)^{\alpha_{3}} h_{3}(w)
$$

with $h_{2} \in C^{0}\left(E \backslash\left\{t_{1}, t_{3}\right\}\right), \quad h_{3} \in C^{0}\left(E \backslash\left\{t_{1}, t_{2}\right\}\right), \quad h_{2}\left(t_{2}\right) \neq 0$, and $h_{3}\left(t_{3}\right) \neq 0$. Since $\psi(w) \in \partial B_{1}$ with $\arg (\psi(w)) \in[\pi / 2,3 \pi / 2]$ for $w \in \sigma_{3} \cup \sigma_{4}, \psi\left(t_{2}\right)=i$, and $\psi\left(t_{3}\right)=-i$, we see that

$$
\begin{aligned}
& |\psi(w)-i|^{-\alpha_{2}} f(w)=\left(\frac{\psi(w)-i}{|\psi(w)-i|}\right)^{\alpha_{2}} h_{2}(w) \rightarrow e^{-\alpha_{2} \pi i} h_{2}\left(t_{2}\right) \quad \text { as } \sigma_{3} \ni w \rightarrow t_{2}, \\
& |\psi(w)+i|^{-\alpha_{3}} f(w)=\left(\frac{\psi(w)+i}{|\psi(w)+i|}\right)^{\alpha_{3}} h_{3}(w) \rightarrow e^{\alpha_{3} \pi i} h_{3}\left(t_{3}\right) \quad \text { as } \sigma_{4} \ni w \rightarrow t_{3} .
\end{aligned}
$$


Since $\operatorname{Re}(-f(w))=0$ if $w \in\left(\sigma_{3} \cup \sigma_{4}\right) \backslash\left\{t_{2}, t_{3}\right\}$ and so $\operatorname{Re}\left(|\psi(w)-i|^{-\alpha_{2}} f(w)\right)=0$ and $\operatorname{Re}\left(|\psi(w)+i|^{-\alpha_{3}} f(w)\right)=0$ for $w \in\left(\sigma_{3} \cup \sigma_{4}\right) \backslash\left\{t_{2}, t_{3}\right\}$, we see that

$$
h_{2}\left(t_{2}\right)=i \lambda_{2} e^{\alpha_{2} \pi i} \quad \text { and } \quad h_{3}\left(t_{3}\right)=i \lambda_{3} e^{-\alpha_{3} \pi i}
$$

for some $\lambda_{2}, \lambda_{3} \in \mathbb{R}$. Since $\operatorname{Im}(f)>0$ on $\left(\sigma_{3} \cup \sigma_{4}\right) \backslash\left\{t_{2}, t_{3}\right\}$, we have $\lambda_{2}, \lambda_{3}>0$,

$$
\begin{array}{ll}
f(w)=(\psi(w)-i)^{\alpha_{2}} i \lambda_{2} e^{\alpha_{2} \pi i}+o\left(|\psi(w)-i|^{\alpha_{2}}\right) & \text { for } w \in E \text { near } t_{2}, \\
f(w)=(\psi(w)+i)^{\alpha_{3}} i \lambda_{3} e^{-\alpha_{3} \pi i}+o\left(|\psi(w)+i|^{\alpha_{3}}\right) & \text { for } w \in E \text { near } t_{3} .
\end{array}
$$

Recall that $w_{(1)}(\tau)=w_{1}+r_{1} e^{\tau i}$ and define

$$
\begin{array}{ll}
u_{(1)}(\tau)=\operatorname{Re}\left(w_{(1)}(\tau)\right), & x_{1}(\tau)=x\left(u_{(1)}(\tau), v_{(1)}(\tau)\right), \\
v_{(1)}(\tau)=\operatorname{Im}\left(w_{(1)}(\tau)\right), & y_{1}(\tau)=y\left(u_{(1)}(\tau), v_{(1)}(\tau)\right) .
\end{array}
$$

Also recall $w_{(2)}(\tau)=w_{2}+r_{2} e^{\tau i}$ and define

$$
\begin{array}{ll}
u_{(2)}(\tau)=\operatorname{Re}\left(w_{(2)}(\tau)\right), & x_{2}(\tau)=x\left(u_{(2)}(\tau), v_{(2)}(\tau)\right), \\
v_{(2)}(\tau)=\operatorname{Im}\left(w_{(2)}(\tau)\right), & y_{2}(\tau)=y\left(u_{(2)}(\tau), v_{(2)}(\tau)\right) .
\end{array}
$$

Recall that the condition $\operatorname{Re}[G(w) f(w)]=0$

for $w \in \sigma_{1} \backslash\left\{t_{1}, t_{2}\right\}$ implies $y_{1}^{\prime}(\tau)=\cot (\delta) x_{1}^{\prime}(\tau)$ for $\tau \in\left(\tau_{1 B}, \tau_{1 A}\right)$ and

for $w \in \sigma_{2} \backslash\left\{t_{1}, t_{3}\right\}$ implies $y_{1}^{\prime}(\tau)=\tan (\delta) x_{1}^{\prime}(\tau)$ for $\tau \in\left(\tau_{1 B}, \tau_{1 A}\right)$.

Thus $K\left(w_{(1)}(\tau)\right)$ maps $\left[\tau_{1 B}, \tau_{1 A}\right]$ onto a line segment on the line $y=\cot (\delta) x+1$ (with $\left.K\left(w_{(1)}\left(\tau_{1 B}\right)\right)=K\left(t_{2}\right)=(0,1)\right)$ and $K\left(w_{(2)}(\tau)\right)$ maps $\left[\tau_{2 A}, \tau_{2 B}\right]$ onto a line segment on the line $y=\tan (\delta) x+y_{0}$ for some undetermined $y_{0}$.

Do we know that $K$ maps $\partial E$ onto a nonconvex quadrilateral of the type desired? Actually, the answer is no. Figure 4 represents the types of nonconvex quadrilaterals onto which $K$ could map $\partial E$. We need to show that Figure 4(C) corresponds to the actual image of $K$. (Different choices of $\alpha_{1}, \alpha_{2}$ and $\alpha_{3}$ do yield all of the possibilities illustrated in (A), (B), and (C) of Figure 4.)

Since $\psi(w) \in \partial B_{1}$ with $\arg (\psi(w)) \in(0, \pi / 2)$ when $w \in \sigma_{1} \backslash\left\{t_{1}, t_{2}\right\}$, we see that

$$
\left|\psi\left(w_{(1)}(\tau)\right)-i\right|^{-\alpha_{2}} f\left(w_{(1)}(\tau)\right)=\left(\frac{\psi\left(w_{(1)}(\tau)\right)-i}{\left|\psi\left(w_{(1)}(\tau)\right)-i\right|}\right)^{\alpha_{2}} h_{2}\left(w_{(1)}(\tau)\right) \rightarrow h_{2}\left(t_{2}\right)
$$

as $\tau \rightarrow \tau_{1 B}^{+}$. Notice that

$$
\begin{aligned}
& \frac{d}{d \tau}\left(x_{1}(\tau)\right)=\frac{-r_{1} \sin (\tau)}{v_{(1)}(\tau)-v_{1}} \operatorname{Im}\left(\left(w_{(1)}(\tau)-w_{1}\right) f\left(w_{(1)}(\tau)\right)\left(1-w_{(1)}^{2}(\tau)\right)\right), \\
& \frac{d}{d \tau}\left(y_{1}(\tau)\right)=\frac{-r_{1} \sin (\tau)}{v_{(1)}(\tau)-v_{1}} \operatorname{Im}\left(\left(w_{(1)}(\tau)-w_{1}\right) i f\left(w_{(1)}(\tau)\right)\left(1+w_{(1)}^{2}(\tau)\right)\right) .
\end{aligned}
$$




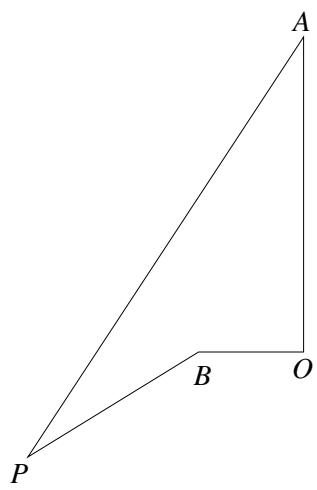

(A)

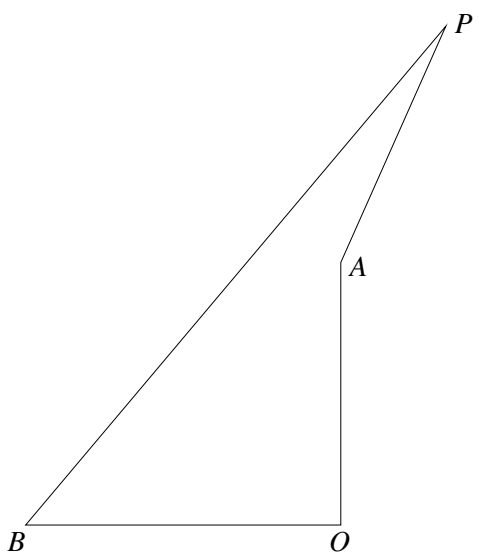

(B)

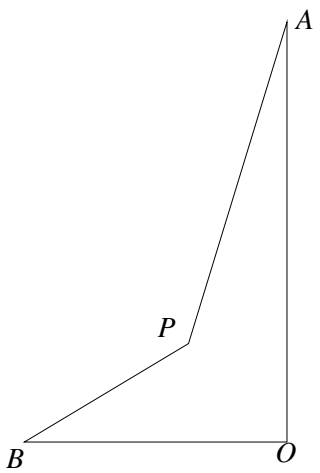

(C)

Figure 4. Types of quadrilaterals onto which $K$ could map $\partial E$.

Recall that $h_{2}\left(t_{2}\right)=i \lambda_{2} e^{\alpha_{2} \pi i}, t_{2}-w_{1}=r_{1} e^{\tau_{1 B} i}, \sin \left(\tau_{1 B}\right)<0, v_{1}>0, \operatorname{Im}\left(t_{2}\right)=0$, and $\alpha_{2} \pi+\tau_{1 B}+\pi / 2=-\left(\lambda_{1}\left(t_{2}\right)+\delta\right) \in(\delta, \pi / 2]$. Hence $\cos \left(\alpha_{2} \pi+\tau_{1 B}+\pi / 2\right) \geq 0$ and $\sin \left(\alpha_{2} \pi+\tau_{1 B}+\pi / 2\right)>0$. Now

$$
\begin{aligned}
\lim _{\tau \rightarrow \tau_{1 B}^{+}} \frac{\left|\psi\left(w_{1}(\tau)\right)-i\right|^{-\alpha_{2}}}{\left|1-w_{(1)}^{2}(\tau)\right|} \frac{d}{d \tau}\left(x_{1}(\tau)\right) & =\frac{-r_{1} \sin \left(\tau_{1 B}\right)}{0-v_{1}} \operatorname{Im}\left(\left(t_{2}-w_{1}\right) i \lambda_{2} e^{\alpha_{2} i}\right) \\
& =\frac{-r_{1} \sin \left(\tau_{1 B}\right)}{-v_{1}} r_{1} \lambda_{2} \sin \left(\alpha_{2} \pi+\tau_{1 B}+\frac{\pi}{2}\right)<0,
\end{aligned}
$$

$\lim _{\tau \rightarrow \tau_{1 B}^{+}}\left|\psi\left(w_{1}(\tau)\right)-i\right|^{-\alpha_{2}} \frac{d}{d \tau}\left(y_{1}(\tau)\right)=\frac{-r_{1} \sin \left(\tau_{1 B}\right)}{0-v_{1}}\left(1+t_{2}^{2}\right) \operatorname{Im}\left(\left(t_{2}-w_{1}\right) i^{2} \lambda_{2} e^{\alpha_{2} i}\right)$

$$
=\frac{-r_{1} \sin \left(\tau_{1 B}\right)}{-v_{1}} r_{1} \lambda_{2}\left(1+t_{2}^{2}\right) \cos \left(\alpha_{2} \pi+\tau_{1 B}+\frac{\pi}{2}\right) \leq 0 .
$$

These inequalities imply that there exists an $\epsilon>0$ such that $x_{1}^{\prime}(\tau)<0$ and, if $\gamma_{1}>\delta$ (so $\left.\tau_{1 B}>-\pi / 2\right), y_{1}^{\prime}(\tau)<0$ for $\tau \in\left(\tau_{1 B}, \tau_{1 B}+\epsilon\right)$.

We leave it to the reader to verify that if $\tau_{0}$ is in $\left(\tau_{1 B}, \tau_{1 A}\right)$ and $x_{1}^{\prime}\left(\tau_{0}\right)=0$, then $f\left(w_{(1)}\left(\tau_{0}\right)\right)=0$; this would use that $\left|w_{(1)}\left(\tau_{0}\right)\right|<1$. Since $f \neq 0$ on $\sigma_{1} \backslash\left\{t_{1}, t_{2}\right\}$, we see that $x_{1}^{\prime}(\tau) \neq 0$ and $y_{1}^{\prime}(\tau) \neq 0$ if $\tau \in\left(\tau_{1 B}, \tau_{1 A}\right)$. Therefore,

$$
x_{1}^{\prime}(\tau)<0 \quad \text { and } \quad y_{1}^{\prime}(\tau)<0 \quad \text { for } \tau \in\left(\tau_{1 B}, \tau_{1 A}\right)
$$

and $K$ is a homeomorphism from $\sigma_{1}$ onto the line segment between $A=(0,1)$ $\left(=K\left(t_{2}\right)\right)$ and $P=\left(x_{P}, y_{P}\right)$, for some $x_{P}<0$ and $y_{P}=\cot (\delta) x_{P}+1 . K\left(\sigma_{1}\right)$ is a subset of the line $y=\cot (\delta) x+1$. 
Notice that

$$
\begin{aligned}
& \frac{d}{d \tau}\left(x_{2}(\tau)\right)=\frac{-r_{2} \cos (\tau)}{u_{(2)}(\tau)-u_{2}} \operatorname{Im}\left(\left(w_{(2)}(\tau)-w_{2}\right) f\left(w_{(2)}(\tau)\right)\left(1-w_{(2)}^{2}(\tau)\right)\right), \\
& \frac{d}{d \tau}\left(y_{2}(\tau)\right)=\frac{-r_{2} \cos (\tau)}{u_{(2)}(\tau)-u_{2}} \operatorname{Im}\left[\left(w_{(2)}(\tau)-w_{2}\right) i f\left(w_{(2)}(\tau)\right)\left(1+w_{(2)}^{2}(\tau)\right)\right] .
\end{aligned}
$$

Arguing as above, we find that

$\lim _{\tau \rightarrow \tau_{2 B}^{-}}\left|\psi\left(w_{2}(\tau)\right)+i\right|^{-\alpha_{3}} x_{2}^{\prime}(\tau)=\frac{-r_{2} \cos \left(\tau_{2 B}\right)}{0-u_{2}} r_{2} \lambda_{3}\left(1-t_{3}^{2}\right) \sin \left(\frac{\pi}{2}+\tau_{2 B}-\alpha_{2} \pi\right) \leq 0$,

$\lim _{\tau \rightarrow \tau_{2 B}^{-}} \frac{\left|\psi\left(w_{2}(\tau)\right)+i\right|^{-\alpha_{3}}}{\left|1-w_{(2)}^{2}(\tau)\right|} y_{1}^{\prime}(\tau)=\frac{-r_{2} \cos \left(\tau_{2 B}\right)}{0-u_{2}} r_{2} \lambda_{3} \cos \left(\frac{\pi}{2}+\tau_{2 B}-\alpha_{2} \pi\right)<0$,

since $\tau_{2 B}+\pi / 2-\alpha_{2} \pi=\pi / 2-\left(\lambda_{2}\left(t_{3}\right)-\delta\right) \in[0, \pi / 2-\delta)$. Hence $y_{2}^{\prime}(\tau)=$ $\tan (\delta) x_{2}^{\prime}(\tau)<0$ for $\tau \in\left(\tau_{2 A}, \tau_{2 B}\right)$. Since $y_{2}\left(\tau_{2 B}\right)=\operatorname{Im}\left(K\left(t_{3}\right)\right)=0, \tau_{2 A}<\tau_{2 B}$, and $y_{2}(\tau)$ is a decreasing function of $\tau$, we have $y_{2}\left(\tau_{2 A}\right)>0$. This implies $x_{B}=$ $\operatorname{Re}\left(K\left(t_{3}\right)\right)=x_{2}\left(\tau_{2 B}\right)<x_{P}$. Therefore $K$ maps $\partial E$ homeomorphically onto the boundary of a nonconvex quadrilateral $A P B O$ with $K$ mapping $\sigma_{1}$ onto $A P, \sigma_{2}$ onto $P B, \sigma_{3}$ onto $B O$, and $\sigma_{4}$ onto $O A$.

Each point $w \in E_{0}=E \backslash \partial E$ lies in the open disc $B_{1}$, and so the tangent plane to $X\left(E_{0}\right)$ at $X(w)$ is not vertical (for example, (13)). Hence $w$ has an open neighborhood $U$ whose closure is strictly contained in $B_{1}$ and whose image $X(U)$ under $X$ is a $C^{2}$ function over an open set in the $x y$-plane. Since the boundary $X(\partial E)$ has a simple projection onto the quadrilateral $A P B O$, it follows that $X(E)$ is the graph of a function in $h \in C^{2}(\Omega(B)) \cap C^{0}(\overline{\Omega(B)})$ over the $x y$-plane; see for example [Huff and McCuan 2006, Lemma 1].

If $\gamma_{1}+\gamma_{2}>\pi / 2-2 \delta, \gamma_{1}>\delta$, and $\gamma_{2}>\delta$, then the stereographic projection of the image of the Gauss map of $X(E)$ is contained in a compact subset of the open unit disk $B_{1}$, and hence $\|\nabla h\|$ is uniformly bounded in $\Omega$. From this, it follows that $h \in C^{1}(\bar{\Omega})$.

Suppose there exists another value $x_{1} \in(-\cot (\delta),-\tan (\delta))$ such that if $B_{1}=$ $\left(x_{1}, 0\right), \Omega=\Omega\left(B_{1}\right)$, and $\Omega_{0}=\Omega_{0}\left(B_{1}\right)$, then the boundary value problem (1) with $\kappa=\lambda=0$ and $\gamma$ as defined in Section 1 has a solution $h \in C^{2}(\Omega) \cap C^{0}(\bar{\Omega})$ which satisfies the conditions that $h(0,0)=0$ and whose Gauss map $\tilde{G}$ over $\Omega_{0}$, given in (5), extends to a map $\tilde{G} \in C^{0}\left(\overline{\Gamma_{0}}: \mathbf{S}^{2}\right)$ which is injective. From the symmetry of the problem, the comparison principle implies that $h=0$ on $O A$ and on $O B$. Suppose $X: E \rightarrow \mathbb{R}^{3}, X(w)=(x(w), y(w), z(w))$, is an isothermal parametrization of $S_{0}$, the graph of $h$ over $\Omega_{0}$ which maps $t_{1}$ to $(P, h(P)), t_{2}$ to $(A, h(A))$, and $t_{3}$ to $(B, h(B))$. Then it follows from the symmetries in the capillary problem and the symmetries of $E$ that $X$ maps 0 to $(0,0,0)$. If we define $\tilde{g}: E \rightarrow \mathbb{C}$ by $\tilde{g}=p \circ \tilde{G} \circ X$, where $p\left(x_{1}, x_{2}, x_{3}\right)=\left(x_{1}+i x_{2}\right) /\left(1-x_{3}\right)$ is the usual stereographic projection, then 
$\tilde{g}$ is an analytic function; see for example [Osserman 1986]. Notice that Section 2 implies $\tilde{g}: \partial E \rightarrow \partial E, \tilde{g}\left(t_{1}\right)=t_{1}, \tilde{g}\left(t_{2}\right)=t_{2}$, and $\tilde{g}\left(t_{3}\right)=t_{3}$. Since $\tilde{G}$ is injective on $\overline{\Gamma_{0}}, \tilde{g}$ is injective, and so $\tilde{g}$ must be a conformal map from $E$ to $E$ that equals the identity; that is, $\tilde{g}=g$. Let us define the analytic function $f: E \rightarrow \mathbb{C}$ by $f(w)=x_{w}(w)-i y_{w}(w)$. From Section 2, we see that $f$ must be a solution of the Hilbert problem (10) and must therefore be the one obtained earlier here. However, this implies $x_{1}=x_{B}\left(=\operatorname{Re}\left(K\left(t_{3}\right)\right)\right)$.

\section{Proof of Theorem 3.2}

Suppose next that $\gamma_{1}+\gamma_{2}<\frac{\pi}{2}-2 \delta$, so that $L=4$. Then

$$
\begin{aligned}
& t_{1}=e^{\left(\pi-\delta-\gamma_{1}\right) i}=w_{1}+r_{1} e^{\left(\pi / 2-\gamma_{1}-\delta\right) i}, t_{3}=w_{2}+r_{2} e^{i \tau_{2 B}} \text { for some } \tau_{2 B} \in\left(\delta-\frac{\pi}{2}, 0\right], \\
& t_{4}=e^{\left(\pi / 2+\delta+\gamma_{2}\right) i}=w_{2}+r_{2} e^{\left(\gamma_{2}+\delta-\pi\right) i}, t_{2}=w_{1}+r_{1} e^{i \tau_{1 B}} \text { for some } \tau_{1 B} \in\left[-\frac{\pi}{2},-\delta\right) .
\end{aligned}
$$

For the sake of definiteness, we set

$$
\begin{aligned}
& \arg \left(G_{1}\left(t_{1}-0\right)\right)=\pi-4\left(\pi-\delta-\gamma_{1}\right), \\
& \arg \left(G_{1}\left(t_{1}+0\right)\right)=-2\left(\delta+\left(\pi / 2-\gamma_{1}-\delta\right)+\left(\pi / 2-\gamma_{1}-2 \delta\right)\right), \\
& \arg \left(G_{1}\left(t_{2}-0\right)\right)=-2\left(\delta+\tau_{1 B}+\lambda_{1}\left(t_{2}\right)\right), \\
& \arg \left(G_{1}\left(t_{2}+0\right)\right)=\arg \left(G_{1}\left(t_{3}-0\right)\right)=\pi, \\
& \arg \left(G_{1}\left(t_{3}+0\right)\right)=\pi+2 \delta-2\left(\tau_{2 B}+\lambda_{2}\left(t_{3}\right)\right), \\
& \arg \left(G_{1}\left(t_{4}-0\right)\right)=\pi+2 \delta-2\left(\left(\gamma_{2}+\delta-\pi\right)+\left(2 \delta+\gamma_{2}-\pi / 2\right)\right), \\
& \arg \left(G_{1}\left(t_{4}+0\right)\right)=\pi-4\left(\pi / 2+\delta+\gamma_{2}\right) .
\end{aligned}
$$

Then

$$
\begin{array}{ll}
\theta_{1}=-1 / 2, & \theta_{2}=-1 / 2-\left(\delta+\lambda_{1}\left(t_{2}\right)+\tau_{1 B}\right) / \pi \in(-1 / 2+2 \delta / \pi, 1 / 2], \\
\theta_{4}=5 / 2, & \theta_{3}=\left(\lambda_{2}\left(t_{3}\right)+\tau_{2 B}-\delta\right) / \pi \in(-1 / 2+2 \delta / \pi, 1 / 2] .
\end{array}
$$

Also $\theta_{1}+\theta_{2}+\theta_{3}+\theta_{4} \in(1+4 \delta / \pi, 3]$.

Let us set $v_{1}=-1, v_{2}=0, v_{3}=0, v_{4}=2, \alpha_{1}=\theta_{1}+1, \alpha_{2}=\theta_{2}, \alpha_{3}=\theta_{3}$, and $\alpha_{4}=\theta_{4}-2$. Let $\psi$ be the conformal map from $E$ onto the unit disk $B_{1}$ which maps $t_{1}$ to $1, t_{2}$ to $i$, and $t_{3}$ to $-i$. Set $\psi\left(t_{4}\right)=\zeta_{4}$. Define $G^{*}: \partial B_{1} \rightarrow \mathbb{C}$ by

$$
G^{*}(\zeta)=G\left(\psi^{-1}(\zeta)\right) \text { for } \zeta \in \partial B_{1} .
$$

Our Hilbert problem (10) is then equivalent to finding $f^{*} \in C^{0}\left(\overline{B_{1}} \backslash\left\{1, \pm i, \zeta_{4}\right\}\right)$ which is analytic in $B_{1}$, has (at worst) integrable singularities at $1, i,-i, \zeta_{4}$, and satisfies

$$
\operatorname{Re}\left(G^{*}(\zeta) f^{*}(\zeta)\right)=0 \quad \text { for } \zeta \in \partial B_{1} \backslash\left\{1, \pm i, \zeta_{4} \cdot\right\}
$$




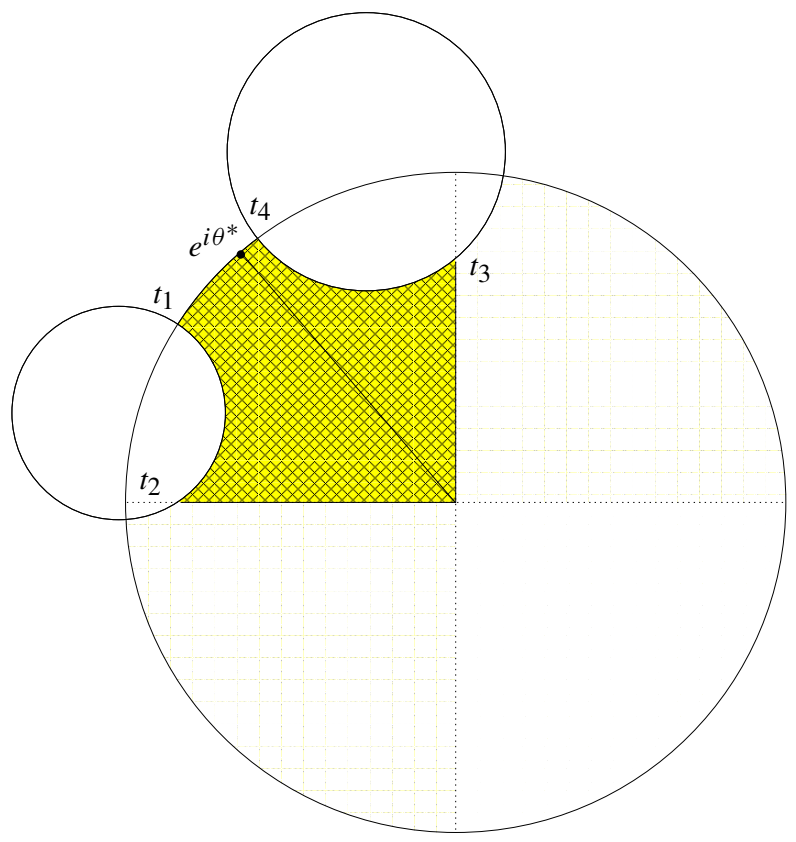

Figure 5. Region $E$ for Theorem 3.2.

Since $v_{1}+v_{2}+v_{3}+v_{4}=1$, this Hilbert problem has a solution of the form

$$
f_{\mu}^{*}(\zeta)=c\left(e^{i \mu} \zeta-e^{-i \mu}\right) \Pi(\zeta) e^{\Gamma_{0}(\zeta)}
$$

where $c, \mu \in \mathbb{R}$ and $\Pi(\zeta)=(\zeta-1)^{\alpha_{1}}(\zeta-i)^{\alpha_{2}}(\zeta+i)^{\alpha_{3}}\left(\zeta-\zeta_{4}\right)^{\alpha_{4}}$. Recall that $e^{\Gamma_{0}(\zeta)}$ is nonvanishing and continuous on $\overline{B_{1}}$.

Now select $\theta^{*} \in\left[\pi / 2+\delta+\gamma_{2}, \pi-\delta-\gamma_{1}\right]$, and note that $e^{i \theta^{*}} \in \sigma_{5}$; see Figure 5. Set

$$
\mu=-\arg \left(\psi\left(e^{i \theta^{*}}\right)\right) / 2 \in\left[0,-\arg \left(\zeta_{4}\right) / 2\right],
$$

so that $e^{-2 i \mu}=\psi\left(e^{i \theta^{*}}\right)$. With this choice, we see that

$$
\begin{aligned}
& f_{\mu}^{*}(\zeta)=c e^{i \mu}\left(\zeta-e^{-2 \mu i}\right) \Pi(\zeta) e^{\Gamma_{0}(\zeta)} \\
& f_{\mu}(w)=c e^{i \mu}\left(\psi(w)-e^{-2 \mu i}\right) \Pi(\psi(w)) e^{\Gamma_{0}(\psi(w))} .
\end{aligned}
$$

Let $c \in \mathbb{R}$ be given by (12), with the obvious interpretation of $f_{0}(w)$. If we define $p(\zeta)=2 c G^{*}(\zeta) e^{i \mu} \Pi(\zeta) e^{\Gamma_{0}(\zeta)}$, then we see $p\left(e^{-2 \mu i}\right) \neq 0$, and so

(16) $\lim _{\theta \uparrow-2 \mu} \frac{2 G^{*}\left(e^{i \theta}\right) f_{\mu}^{*}\left(e^{i \theta}\right)}{\left|e^{i \theta}-e^{-2 i \mu}\right|}=\lim _{\theta \uparrow-2 \mu} p\left(e^{i \theta}\right) \frac{e^{i \theta}-e^{-2 i \mu}}{\left|e^{i \theta}-e^{-2 i \mu}\right|}=-i e^{-2 i \mu} p\left(e^{-2 \mu i}\right)$ 
and

$$
\lim _{\theta \downarrow-2 \mu} \frac{2 G^{*}\left(e^{i \theta}\right) f_{\mu}^{*}\left(e^{i \theta}\right)}{\left|e^{i \theta}-e^{-2 i \mu}\right|}=\lim _{\theta \downarrow-2 \mu} p\left(e^{i \theta}\right) \frac{e^{i \theta}-e^{-2 i \mu}}{\left|e^{i \theta}-e^{-2 i \mu}\right|}=i e^{-2 i \mu} p\left(e^{-2 \mu i}\right) .
$$

Using (15), we see that $e^{-2 i \mu} p\left(e^{-2 \mu i}\right) \in \mathbb{R} \backslash\{0\}$.

Let $X_{\mu}: E \rightarrow \mathbb{R}^{3}, X_{\mu}(u+i v)=\left(x_{\mu}(u, v), y_{\mu}(u, v), z_{\mu}(u, v)\right)$ be the minimal surface satisfying $X(0)=(0,0,0)$ whose Weierstrass $(f, g)$-representation is given by $f_{\mu}$ and $g(w)=w$. From the arguments in the proof of Theorem 3.1, we see that

(i) there exists $x_{B} \in(-\cot (\delta),-\tan (\delta))$ and $B=\left(x_{B}, 0\right)$ such that $X_{\mu}(\partial E)$ projects onto $\partial(\Omega(B))$,

(ii) $X_{\mu}$ is a homeomorphism from $E$ onto $X_{\mu}(E)$, and

(iii) $K_{\mu}$ maps $\sigma_{1}$ onto $A P, \sigma_{2}$ onto $P B, \sigma_{3}$ onto $B O, \sigma_{4}$ onto $O A$, and $\sigma_{5}$ to the point $P$.

Notice, for example, that the argument in the previous section showing $x_{1}^{\prime}(\tau)<0$, $y_{1}^{\prime}(\tau)<0$ and $x_{2}^{\prime}(\tau)<0, y_{2}^{\prime}(\tau)<0$ remains valid here (with $x_{1}^{\prime}(\tau)<0, y_{1}^{\prime}(\tau)<0$ for $\tau \in\left(\tau_{1 B}, \frac{\pi}{2}-\gamma_{1}-\delta\right)$ and $x_{2}^{\prime}(\tau)<0, y_{2}^{\prime}(\tau)<0$ for $\left.\left.\tau \in\left(\gamma_{2}+\delta-\pi, \tau_{2 B}\right)\right)\right)$.

Notice that a classical "tilting argument" (for example [Huff and McCuan 2006, Section 3]), together with the argument in Section 5, shows that $X\left(E_{0}\right)$ is the graph of a function $h_{\mu} \in C^{2}(\Omega(B))$. Now $h_{\mu}$ is discontinuous at $P$ and the radial limits $R h_{\mu}$ of $h_{\mu}$ at $P$ contain a central fan if $\theta^{*} \in\left(\pi / 2+\delta+\gamma_{2}, \pi-\delta-\gamma_{1}\right)$ with $R h_{\mu}$ constant on $\left[\theta^{*}-3 \pi / 2, \theta^{*}-\pi / 2\right]$.

Since $K_{\mu}(w)=P$ for all $w \in \sigma_{5}$ while $d / d \tau\left(z_{\mu}\left(e^{i \tau}\right)\right) \neq 0$ for $\tau \in\left(\arg \left(\zeta_{4}\right), 0\right)$ with $\tau \neq-2 \mu$, we see that $h_{\mu}$ is discontinuous at $P$ if $\theta^{*} \in\left(\pi / 2+\delta+\gamma_{2}, \pi-\delta-\gamma_{1}\right)$. Suppose now $\theta^{*}$ is in $\left(\pi / 2+\delta+\gamma_{2}, \pi-\delta-\gamma_{1}\right)$. Notice that

$$
\frac{d}{d \tau}\left(z_{\mu}\left(e^{i \tau}\right)\right)=-2 \operatorname{Im}\left(G\left(e^{i \tau}\right) f\left(e^{i \tau}\right)\right)
$$

and, since $e^{-2 i \mu}=\psi\left(e^{i \theta^{*}}\right),(16)$ and (17) show that this derivative changes sign at $\tau=\theta^{*}$. This implies $z_{\mu}\left(e^{i \tau}\right)$ is increasing on one side of $\theta^{*}$ and decreasing on the other side of $\theta^{*}$ for $\tau \in\left(\pi / 2+\delta+\gamma_{2}, \pi-\delta-\gamma_{1}\right)$. Set

$$
\xi_{1}=\delta+\gamma_{2}-\pi, \quad \xi_{L}=\theta^{*}-3 \pi / 2, \quad \xi_{R}=\theta^{*}-\pi / 2, \quad \xi_{2}=\pi / 2-\delta-\gamma_{1} .
$$

From our construction, $X_{\mu}$ has a (boundary) branch point at $e^{i \theta^{*}}$. From (13), we see that $\vec{N}\left(e^{i \theta^{*}}\right)=\left(\cos \left(\theta^{*}\right), \sin \left(\theta^{*}\right), 0\right)$. Let $D=\left\{u+i v \in \mathbb{C}: u^{2}+v^{2}<1, v>0\right\}$, and let $\chi$ be a conformal map from $D$ to $E_{0}$ which maps 0 to $e^{i \theta^{*}}$. Let $X: D \rightarrow \mathbb{R}^{3}$ be defined by $X=X_{\mu} \circ \chi$, and let $X(u+i v)=(x(u, v), y(u, v), z(u, v))$ denote the components of $X$. From [Lancaster and Siegel 1996b, p. 174], we see that

$$
x_{v}(u, 0)=\sin \left(\theta^{*}\right) u+o(|u|) \text { and } y_{v}(u, 0)=-\cos \left(\theta^{*}\right) u+o(|u|) \text { as } u \rightarrow 0 .
$$




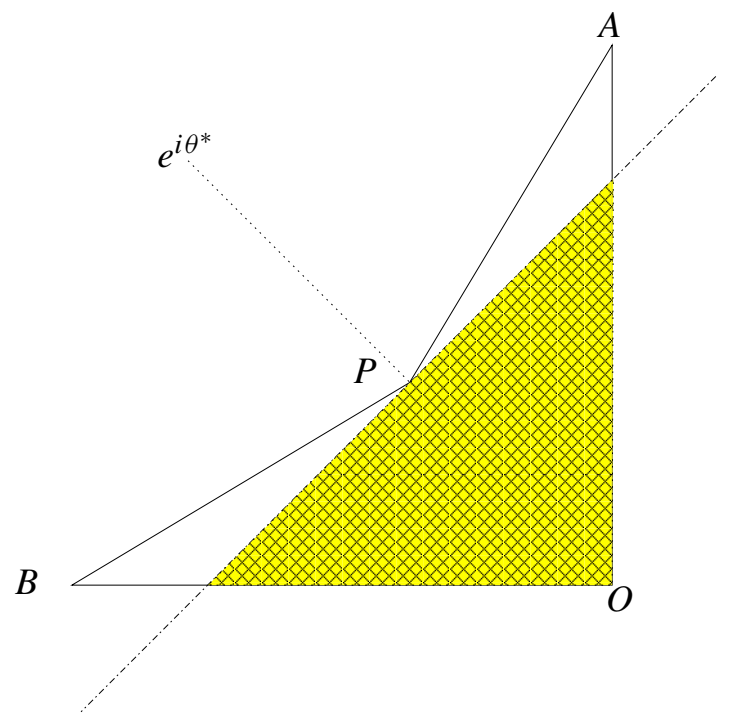

Figure 6. The "central fan region".

Hence

$$
\frac{\left(x_{v}(u, 0), y_{v}(u, 0)\right)}{\sqrt{x_{v}^{2}(u, 0)+y_{v}^{2}(u, 0)}} \rightarrow \begin{cases}\left(\cos \left(\xi_{L}\right), \sin \left(\xi_{L}\right)\right) & \text { as } u \uparrow 0, \\ \left(\cos \left(\xi_{R}\right), \sin \left(\xi_{R}\right)\right) & \text { as } u \downarrow 0 .\end{cases}
$$

As in Steps 2 and 3 of the proof of [Lancaster and Siegel 1996b, Theorem 1(ii)], this implies that the radial limits of $h_{\mu}$ at $P$ satisfy

$$
R h_{\mu}(\theta) \text { is } \begin{cases}\lim _{\tau \rightarrow-2 \mu^{-}} z_{\mu}(\tau) & \text { if } \delta-\pi \leq \theta \leq \xi_{1}, \\ \text { strictly decreasing } & \text { if } \xi_{1}<\theta<\xi_{L}, \\ \text { constant } & \text { if } \xi_{L} \leq \theta \leq \xi_{R}, \\ \text { strictly increasing } & \text { if } \xi_{R}<\theta<\xi_{2}, \\ \lim _{\tau \rightarrow-2 \mu^{+}} z_{\mu}(\tau) & \text { if } \xi_{2} \leq \theta \leq \frac{\pi}{2}-\delta .\end{cases}
$$

The behavior of $R h_{\mu}(\cdot)$ on $\left[\alpha_{L}, \alpha_{R}\right]$ is the definition of a "central fan" of (constant) radial limits (for example [Lancaster and Siegel 1996b]), and the "central fan region" (for example $\left.\left\{(r \cos (\theta), r \sin (\theta)) \in \Omega: r>0, \theta \in\left[\xi_{L}, \xi_{R}\right]\right\}\right)$ is illustrated in Figure 6. If $\theta^{*}=\pi / 2+\delta+\gamma_{2}$, then $\xi_{L}=\xi_{1}, \xi_{L}=\delta+\gamma_{2}$, and $R h_{\mu}(\theta)$ is constant on $\left[\delta-\pi, \xi_{R}\right]$ while if $\theta^{*}=\pi-\delta-\gamma_{1}$, then $\xi_{R}=\xi_{2}, \xi_{L}=-\pi / 2-\delta-\gamma_{1}$, and $R h_{\mu}(\theta)$ is constant on $\left[\xi_{L}, \pi / 2-\delta\right]$. We leave it to the reader to verify the uniqueness of $h$ as in the proof of Theorem 3.1.

\section{General results}

Theorem 7.1. Let $\delta \in(0, \pi / 4)$, and suppose $\gamma_{1}, \gamma_{2} \in(0, \pi / 2)$ satisfy (4) and $\gamma_{1}+\gamma_{2} \geq \pi / 2-2 \delta$. Then there exists a unique $x_{0} \in(-\cot (\delta),-\tan (\delta))$ such 
that if $B=\left(x_{0}, 0\right), \Omega=\Omega(B)$, and $\Omega_{0}=\Omega_{0}(B)$, then the boundary value problem (1) with $\kappa=\lambda=0$ and $\gamma$ as defined in Section 1 has a (unique) solution $h \in$ $C^{2}(\Omega) \cap C^{0}(\bar{\Omega} \backslash\{A, B, C, D\})$ which satisfies the conditions that $h(0,0)=0$ and whose Gauss map over $\Omega_{0}, \tilde{G}: \Gamma_{0} \rightarrow \mathbf{S}^{2}$, given in (5) with $\Gamma_{0}=\{(x, y, h(x, y))$ : $\left.(x, y) \in \Omega_{0}\right\}$, extends to a map $\tilde{G} \in C^{0}\left(\overline{\Gamma_{0}}: \mathbf{S}^{2}\right)$ which is injective. In addition, if $\gamma_{1}+\gamma_{2}>\pi / 2-2 \delta$, then $h \in C^{1}(\bar{\Omega} \backslash\{A, B, C, D\})$. If $\gamma_{1} \geq \delta$, then $C^{0}(\bar{\Omega} \backslash\{B, D\})$, while if $\gamma_{2} \geq \delta$, then $C^{0}(\bar{\Omega} \backslash\{A, C\})$.

Theorem 7.2. Let $\delta \in(0, \pi / 4)$ and $\gamma_{1}, \gamma_{2} \in(0, \pi / 2)$ satisfy (4) and

$$
\gamma_{1}+\gamma_{2}<\pi / 2-2 \delta \text {. }
$$

For each $\theta_{1} \in\left[\delta+\gamma_{2}-\pi,-\pi / 2-\delta-\gamma_{1}\right]$, there exists a unique $x_{0}=x_{0}\left(\theta_{1}\right) \in$ $(-\cot (\delta),-\tan (\delta))$ such that if $B=\left(x_{0}, 0\right), \Omega=\Omega(B)$, and $\Omega_{0}=\Omega_{0}(B)$, then the boundary value problem (1) with $\kappa=\lambda=0$ and $\gamma$ as defined in Section 1 has a (unique) solution $h \in C^{2}(\Omega) \cap C^{0}(\bar{\Omega} \backslash\{A, B, C, D, P, Q, R, S\})$ which satisfies the conditions that $h(0,0)=0$ and whose Gauss map $\tilde{G}$ over $\Omega_{0}$, given in (5), extends to a map $\tilde{G} \in C^{0}\left(\overline{\Gamma_{0}}: \mathbf{S}^{2}\right)$ which is injective. This solution is discontinuous at $P$, $Q, R$, and $S$ and

$$
R h(\theta ; P)= \begin{cases}\text { constant } & \text { if } \theta \in\left[\delta-\pi, \delta+\gamma_{2}-\pi\right], \\ \text { strictly decreasing } & \text { if } \theta \in\left[\delta+\gamma_{2}-\pi, \theta_{1}\right], \\ \text { constant } & \text { if } \theta \in\left[\theta_{1}, \theta_{1}+\pi\right], \\ \text { strictly increasing } & \text { if } \theta \in\left[\theta_{1}+\pi, \pi / 2-\delta-\gamma_{1}\right], \\ \text { constant } & \text { if } \theta \in\left[\pi / 2-\delta-\gamma_{1}, \pi / 2-\delta\right] .\end{cases}
$$

$R h(\theta ; Q), R h(\theta ; R)$, and $R h(\theta ; S)$ in an appropriately similar manner. If $\gamma_{1} \geq \delta$, then $h \in C^{0}(\bar{\Omega} \backslash\{B, D, P, Q, R, S\})$. If $\gamma_{2} \geq \delta$, then $h \in C^{0}(\bar{\Omega} \backslash\{A, C, P, Q, R, S\})$.

The are eight different possible geometries of $E$ represented by Theorems 7.1 and 7.2. For Theorem 7.1, they are tabulated as follows.

$$
\begin{aligned}
& \gamma_{1} \geq \delta \quad \gamma_{2} \geq \delta \quad \gamma_{1}+\gamma_{2} \geq \pi / 2-2 \delta \quad L=3 \quad \text { Figure } 2 \\
& \text { (ii) } \quad \begin{array}{lllll}
\gamma_{1} \geq \delta & \gamma_{2}<\delta & \gamma_{1}+\gamma_{2} \geq \pi / 2-2 \delta & L=4 & \text { Figure 7 }
\end{array} \\
& \text { (iii) } \begin{array}{lllll}
\gamma_{1}<\delta & \gamma_{2} \geq \delta & \gamma_{1}+\gamma_{2} \geq \pi / 2-2 \delta & L=4 & \text { Figure 7 }
\end{array}
\end{aligned}
$$

\begin{tabular}{|c|c|c|c|c|c|}
\hline & $\gamma_{1} \geq \delta$ & $\nu_{2} \geq \delta$ & $\gamma_{1}+\gamma_{2}<\pi / 2-2 \delta$ & $L=$ & \\
\hline & $\gamma_{1}>\delta$ & & $\gamma_{1}+\gamma_{2}<\pi / 2-2 \delta$ & $L=5$ & Figure 8 \\
\hline & & $\geq 0$ & $\gamma_{1}+\gamma_{2}<\pi / 2-2 \delta$ & $L=5$ & re 8 \\
\hline & & $<$ & $1+\gamma_{2}<\pi / 2-2 \delta$ & $L=6$ & Figure \\
\hline
\end{tabular}

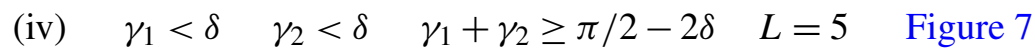

For Theorem 7.2, the table is this:

The results of Theorem 3.1 show that Theorem 7.1 is true in case (i) and Theorem 3.2 shows that Theorem 7.2 is correct in case (v). Here we have used the notation of Section 4 to indicate (by $L$ ) the number of corners, excluding the origin, which 


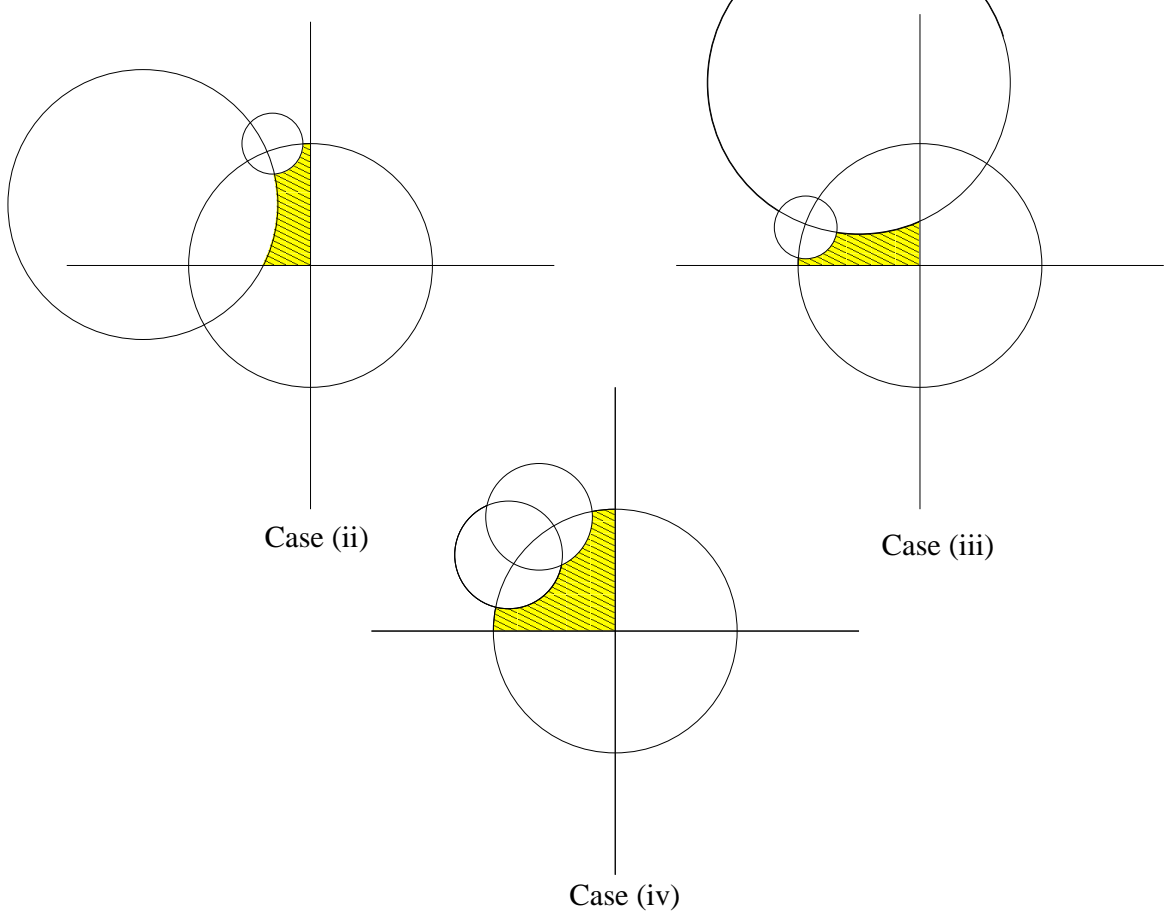

Figure 7. Other Theorem 7.1 cases.

the boundary of $E$ contains. In cases (ii)-(iv), the proof of Theorem 3.1 remains unchanged except for certain details which differ from those in Section 5. The changes for each case are listed below.

(ii) $t_{3}=e^{i\left(\pi / 2+\delta-\gamma_{2}\right)}, t_{4}=i, \theta_{3}=-2 \delta / \pi, \theta_{4}=0$, and $\alpha_{4}=\theta_{4}$.

(iii) $t_{2}=e^{i\left(\pi-\delta+\gamma_{1}\right)}, t_{4}=-1, \theta_{2}=1 / 2+2\left(\delta-\gamma_{1}\right) / \pi, \theta_{4}=0$, and $\alpha_{4}=\theta_{4}$.

(iv) $t_{2}=e^{i\left(\pi-\delta+\gamma_{1}\right)}, t_{3}=e^{i\left(\pi / 2+\delta-\gamma_{2}\right)}, t_{4}=i, t_{5}=-1, \theta_{2}=1 / 2+2\left(\delta-\gamma_{1}\right) / \pi$, $\theta_{3}=-2 \delta / \pi, \theta_{4}=0, \theta_{5}=0, \alpha_{4}=\theta_{4}$, and $\alpha_{5}=\theta_{5}$.

In cases (vi)-(viii), the proof of Theorem 3.2 remains unchanged except for certain differences from Section 6:

(vi) $t_{3}=e^{i\left(\pi / 2+\delta-\gamma_{2}\right)}, t_{5}=i, \theta_{3}=-2 \delta / \pi, \theta_{5}=0$, and $\alpha_{5}=\theta_{5}$.

(vii) $t_{2}=e^{i\left(\pi-\delta+\gamma_{1}\right)}, t_{5}=-1, \theta_{2}=1 / 2+2\left(\delta-\gamma_{1}\right) / \pi, \theta_{5}=0$, and $\alpha_{5}=\theta_{5}$.

(viii) $t_{2}=e^{i\left(\pi-\delta+\gamma_{1}\right)}, t_{3}=e^{i\left(\pi / 2+\delta-\gamma_{2}\right)}, t_{5}=i, t_{6}=-1, \theta_{2}=1 / 2+2\left(\delta-\gamma_{1}\right) / \pi$, $\theta_{3}=-2 \delta / \pi, \theta_{4}=0, \theta_{5}=0, \alpha_{5}=\theta_{5}$, and $\alpha_{6}=\theta_{6}$. 


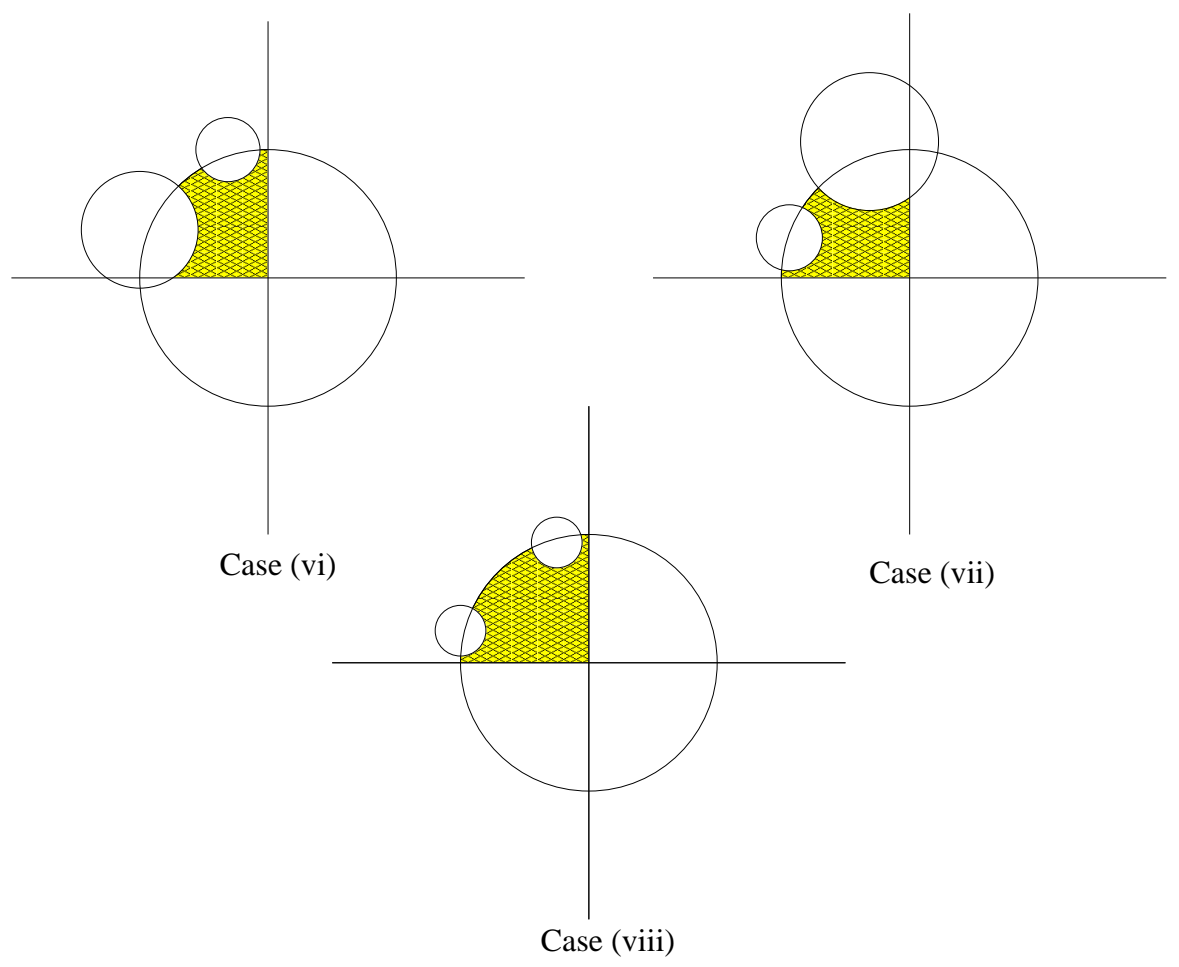

Figure 8. Other Theorem 7.2 cases.

Remark 7.3. It would be interesting to extend this work to appropriate analytic functions $g: E \rightarrow B_{1}$ which map $\partial E$ homeomorphically onto $\partial E$ but are not injective over $E$. In this case, although the surface $z=h(x, y)$ would be a strict minimizer of the appropriate capillary functional, the surface $z=h(x, y)$ would have "undulations" or "wobbles" which one might expect to increase surface area.

\section{Acknowledgment}

The authors are indebted to the Max-Planck-Institut für Mathematik in den Naturwissenschaften, in Leipzig, for its hospitality during the course of the study, especially while the authors attended the Institute's Capillarity Workshop in the summer of 2003, and to the Mathematical Sciences Institute of the Australian National University. The authors also thank Eberhard Zeidler and Ben Andrews for their support and Robert Finn and the referee for their valuable suggestions. 


\section{References}

[Concus and Finn 1974a] P. Concus and R. Finn, "On capillary free surfaces in a gravitational field", Acta Math. 132 (1974), 207-223. MR 58 \#32327c Zbl 0382.76005

[Concus and Finn 1974b] P. Concus and R. Finn, "On capillary free surfaces in the absence of gravity”, Acta Math. 132 (1974), 177-198. MR 58 \#32327a Zbl 0382.76003

[Concus and Finn 1991] P. Concus and R. Finn, "On a comment by J. P. B. Vreeburg”, Microgravity Science and Technology IV (1991), 60.

[Concus and Finn 1994] P. Concus and R. Finn, "Capillary Surfaces in a Wedge — Differing Contact Angles”, Microgravity Science and Technology VII/2 (1994), 152-155.

[Concus and Finn 1996a] P. Concus and R. Finn, "Capillary wedges revisited", SIAM J. Math. Anal. 27:1 (1996), 56-69. MR 96m:76006 Zbl 0843.76012

[Concus and Finn 1996b] P. Concus and R. Finn, "Comments on a paper of J. B. Keller, A. C. King and G. Merchant: "Surface tension"”, Nonlinear Anal. 27:7 (1996), 857-861. MR 99d:76030 Zbl 0869.76016

[Concus et al. 1992] P. Concus, R. Finn, and F. Zabihi, "On canonical cylinder sections for accurate determination of contact angle in microgravity", pp. 125-131 in Fluid mechanics phenomena in microgravity (Anaheim, CA, 1992), edited by D. A. Siginer and M. M. Weislogel, Applied Mechanics Division 154, ASME, New York, 1992.

[Finn 1998] R. Finn, "Featured review of [Lancaster and Siegel 1996b]", Math Reviews, 1998, Available at http://www.ams.org/mathscinet-getitem?mr=1433987.

[Finn 1999] R. Finn, “Capillary surface interfaces”, Notices Amer. Math. Soc. 46:7 (1999), 770-781. MR 2000g:76033

[Finn 2002] R. Finn, "Some properties of capillary surfaces", Milan J. Math. 70 (2002), 1-23. MR 2003m:53012 Zbl 1053.76009

[Huff and McCuan 2006] R. Huff and J. McCuan, "Scherk-type capillary graphs", J. Math. Fluid Mech. 8:1 (2006), 99-119. MR 2006k:76022 Zbl 05058200

[Jin and Lancaster 1999] Z. Jin and K. Lancaster, "Theorems of Phragmén-Lindelöf type for quasilinear elliptic equations", J. Reine Angewante Math. 514 (1999), 165-197. MR 2001c:35074 Zbl 0937.35053

[Keller et al. 1991] J. B. Keller, A. King, and G. Merchant, "Surface tension", pp. 161-168 in Offluid mechanics and related matters (La Jolla, CA, 1990), edited by R. Salmon and D. Betts, Scripps. Inst. Oceanogr. Ref. Ser. 91, Scripps Inst. Oceanogr., San Diego, CA, 1991. MR 99d:76029

[Lancaster 1988] K. E. Lancaster, "Nonparametric minimal surfaces in $\mathbf{R}^{3}$ whose boundaries have a jump discontinuity", Internat. J. Math. and Math. Sci. 11:4 (1988), 651-656. MR 89m:35072 Zbl 0696.35057

[Lancaster $\geq 2008]$ K. E. Lancaster, "Proof of the Concus-Finn conjecture", Preprint.

[Lancaster and Siegel 1996a] K. E. Lancaster and D. Siegel, "Behavior of a bounded non-parametric $H$-surface near a reentrant corner", Z. Anal. Anwendungen 15:4 (1996), 819-850. MR 97m:53011 Zbl 0866.35046

[Lancaster and Siegel 1996b] K. E. Lancaster and D. Siegel, "Existence and behavior of the radial limits of a bounded capillary surface at a corner", Pacific J. Math. 176:1 (1996), 165-194. Correction to figures in 179 (1997), 397-402. MR 98g:58030a Zbl 0866.76018

[Monakhov 1983] V. N. Monakhov, Boundary value problems with free boundaries for elliptic systems of equations, Translations of Mathematical Monographs 57, American Mathematical Society, Providence, RI, 1983. MR 85a:35029 Zbl 0532.35001 
[Osserman 1986] R. Osserman, A survey of minimal surfaces, Second ed., Dover Publications, New York, 1986. MR 87j:53012 Zbl 0209.52901

[Serrin 1969] J. Serrin, "The problem of Dirichlet for quasilinear elliptic differential equations with many independent variables”, Philos. Trans. Roy. Soc. London Ser. A 264 (1969), 413-496. MR 43 \#7772 Zbl 0181.38003

[Shi 2006] D. Shi, “Capillary surfaces at a reentrant corner", Pacific J. Math. 224:2 (2006), 321-353. MR 2008a:58013 Zbl 05163674

[Shi and Finn 2004] D. Shi and R. Finn, "On a theorem of Lancaster and Siegel”, Pacific J. Math. 213:1 (2004), 111-119. MR 2004m:76038 Zbl 02101146

[Tam 1986] L.-F. Tam, "Regularity of capillary surfaces over domains with corners: borderline case", Pacific J. Math. 124:2 (1986), 469-482. MR 87k:49049 Zbl 0604.49029

[Vreeburg 1990] J. P. B. Vreeburg, "Comment on the paper 'Parabolic flight experiments on fluid surfaces and wetting”, Microgravity Science and Technology III (1990), 125.

Received October 23, 2006. Revised October 21, 2007.

MARIA ATHANASSENAS

SCHOOL OF MATHEMATICAL SCIENCES

PO BOX 28M

MONASH UNIVERSITY

Clayton Campus ViC 3800

Australia

maria.athanassenas@sci.monash.edu.au

KIRK LANCASTER

DEPARTMENT OF MATHEMATICS AND STATISTICS

WICHITA STATE UNIVERSITY

WiCHITA, KA 67260-0033

UNITED STATES

lancaster@math.wichita.edu 